\title{
Gauged flavor group with left-right symmetry
}

\author{
Diego Guadagnoli, ${ }^{a}$ Rabindra N. Mohapatra ${ }^{b}$ and Ilmo Sung ${ }^{b}$ \\ ${ }^{a}$ Laboratoire de Physique Théorique, Universite Paris-Sud, \\ Centre d'Orsay, F-91405 Orsay-Cedex, France \\ ${ }^{b}$ Maryland Center for Fundamental Physics, Department of Physics, \\ University of Maryland, College Park, MD 20742, U.S.A. \\ E-mail: diego.guadagnoli@th.u-psud.fr, rmohapat@umd.edu, sung@umd.edu
}

ABSTRACT: We construct an anomaly-free extension of the left-right symmetric model, where the maximal flavor group is gauged and anomaly cancellation is guaranteed by adding new vectorlike fermion states. We address the question of the lowest allowed flavor symmetry scale consistent with data. Because of the mechanism recently pointed out by Grinstein et al. tree-level flavor changing neutral currents turn out to play a very weak constraining role. The same occurs, in our model, for electroweak precision observables. The main constraint turns out to come from $W_{R}$-mediated flavor changing neutral current box diagrams, primarily $K-\bar{K}$ mixing. In the case where discrete parity symmetry is present at the $\mathrm{TeV}$ scale, this constraint implies lower bounds on the mass of vectorlike fermions and flavor bosons of 5 and $10 \mathrm{TeV}$ respectively. However, these limits are weakened under the condition that only $\mathrm{SU}(2)_{R} \times \mathrm{U}(1)_{B-L}$ is restored at the TeV scale, but not parity. For example, assuming the $\mathrm{SU}(2)$ gauge couplings in the ratio $g_{R} / g_{L} \approx 0.7$ allows the above limits to go down by half for both vectorlike fermions and flavor bosons. Our model provides a framework for accommodating neutrino masses and, in the parity symmetric case, provides a solution to the strong $\mathrm{CP}$ problem. The bound on the lepton flavor gauging scale is somewhat stronger, because of Big Bang Nucleosynthesis constraints. We argue, however, that the applicability of these constraints depends on the mechanism at work for the generation of neutrino masses.

Keywords: Beyond Standard Model, Quark Masses and SM Parameters

ARXIV EPRINT: 1103.4170 


\section{Contents}

1 Introduction 1

2 Flavor symmetry within left-right models 3

3 Phenomenology $\quad 7$

3.1 Tree-level FCNC effects 8

3.2 Loop FCNC effects and lower bound on the $W_{R}$ mass 9

$\begin{array}{lll}3.3 & \text { Flavor gauge boson mass scale } & 11\end{array}$

$\begin{array}{lll}3.4 & \text { Fermion mixing and its consequences } & 12\end{array}$

$\begin{array}{lll}3.4 .1 & \text { Electroweak precision tests } & 15\end{array}$

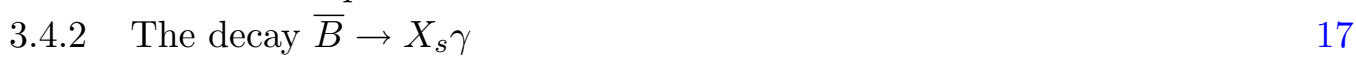

$\begin{array}{llr}3.5 & \text { Further constraints } & 18\end{array}$

$\begin{array}{lll}3.5 .1 & \text { Electric dipole moments } & 18\end{array}$

$\begin{array}{lll}3.5 .2 & \text { Top quark flavor changing effects } & 18\end{array}$

$\begin{array}{lll}3.5 .3 & \text { Direct searches } & 19\end{array}$

4 Lepton sector $\quad 19$

$\begin{array}{lll}4.1 & \text { Neutrino masses } & 19\end{array}$

$\begin{array}{lll}4.2 & \text { Constraints } 20\end{array}$

5 Conclusions $\quad 21$

\section{Introduction}

One of the long-standing mysteries of physics beyond the Standard Model is the origin of flavor patterns for quarks and leptons. In the Standard Model (SM), they arise from the quark and lepton Yukawa couplings with the SM Higgs boson and are arbitrary, thereby precluding any physical insight as to their origin. Since these flavor patterns may well be the remnants of the breaking of some symmetry, the belief is that pinning down the flavor symmetry at work could provide hints of the underlying dynamics at work. Many possibilities for approaching this important issue from the vantage point of symmetry then present themselves - starting from discrete non-abelian subgroups of these flavor symmetries to continuous global or local ones. The question then arises as to how we determine by low energy observations which particular mechanism is at work and at which scale such a symmetry manifests itself. The hope is that different choices will lead to different characteristic predictions, e.g. a global horizontal symmetry would lead to massless familons at low energies [1] and discrete symmetries could lead to some relations between observables.

A widely discussed possibility is to study gauged flavor symmetries [2-9], which leads to a number of interesting effects such as new gauge bosons and new flavor changing effects 
mediated by these bosons. The very first test of this possibility is to determine the scale of gauged flavor symmetry. Naïve considerations seem to suggest that this scale is likely to be in the $1000 \mathrm{TeV}$ range; however in specific models this expectation could change drastically. For example, in a recent paper by Grinstein, Redi and Villadoro (GRV) [10], it has been shown by explicit construction that there are SM extensions with gauged flavor symmetry where this scale could be in the $1 \mathrm{TeV}$ range or even below, compatible with constraints from the hadronic sector. Furthermore this model predicts, by the requirement of anomaly cancellation, new vectorlike quarks, the lightest of which with masses again in the $\mathrm{TeV}$ ballpark, hence within the reach of LHC direct searches. The mechanism at work in the GRV model is an inverse, see-saw like, relation between the masses of the quarks and those of the new fermion states [11-13] as well as of the flavor gauge bosons, so that the partners of the heaviest quarks are the lightest among the new states. This fact allows to pass all the flavor changing neutral current (FCNC) constraints in a very natural way.

A certain degree of model dependence in the idea of gauging the flavor symmetry is the choice of new fermionic states added in order to cancel the gauge anomalies. To achieve this, the simplest option is to include new, vectorlike quark partners. This choice may however appear to be at odds with that of quark states that need sit in chiral representations. This simple consideration motivates us to pursue here an alternative possibility, where the maximal gauging of flavor symmetry is carried out within a left-right symmetric extension of the SM [14-17]. This implies that the flavor symmetry group is itself left-right symmetric. The new gauge anomalies resulting from the larger gauge group cancel with the introduction of vectorlike new fermionic partners of the quarks [11-13]. Besides the known virtues inherent in left-right symmetric extensions of the SM, e.g. the possibility to justify the hypercharge quantum numbers, there appear to be the following advantages in our approach: (i) it provides a natural way to include neutrino masses; (ii) in the quark Lagrangian, it features only three free parameters in the gauge and Yukawa sector, after the rest are fixed by data on quark masses and mixings; (iii) the model provides a simple solution to the strong CP problem without the need for an axion, in a manner similar to that discussed in refs. [18-20].

There are two possible realizations of this idea while keeping the gauge group to be $\mathrm{SU}(2)_{L} \times \mathrm{SU}(2)_{R} \times \mathrm{U}(1)_{B-L}$ at the TeV scale: (a) the discrete parity symmetry is maintained down to the $\mathrm{TeV}$ scale or (b) it is broken at some very high scale [21] so that, at the $\mathrm{TeV}$ scale, the two gauge couplings as well as the left and right Yukawa couplings are in general different from each other. We will consider both alternatives below. Within the second alternative, the solution to the strong CP problem mentioned above is not obvious.

Concerning the constraints on the model outlined above, we find that, similarly as in the GRV model, tree-level FCNCs mediated by the flavor gauge bosons are tamed automatically by the hierarchy of their masses. Also, in our case, electroweak (EW) precision tests are automatically fulfilled in the bulk of the parameter space.

The strongest constraint comes from $W_{R}$-mediated FCNC box diagrams, primarily $K-\bar{K}$ mixing. The implied bounds on the scale of the new vectorlike quarks as well as on the flavor gauge bosons, which will be discussed in detail in sections 3.2, 3.3 and 3.4, depend however on the scale at which parity is broken.

Compatibly with these bounds, the new effects, accessible at the current generation 
of TeV hadron colliders, include the lightest among the new particles' masses and various deviations in top-physics observables, like top production and decays. Such deviations are due to the fact that the top (in particular, the right-handed one) mixes non-negligibly onto its new fermionic partner, whereas mixing is tiny to absent for the rest of the quark states. This in turn explains why no deviations are to be expected in the production or decays of any other quark than $t_{R}$.

For an overview of the organization of this paper we refer the reader to the table of content on page 1.

\section{Flavor symmetry within left-right models}

In the SM, once the Yukawa couplings are set to zero, the maximal flavor symmetry group is $\mathrm{SU}(3)_{Q_{L}} \times \mathrm{SU}(3)_{u_{R}} \times \mathrm{SU}(3)_{d_{R}} \times \mathrm{SU}(3)_{\ell_{L}} \times \mathrm{SU}(3)_{\ell_{R}}$. If the weak gauge group is extended to that of the left-right symmetric model, the flavor group becomes $\mathrm{SU}(3)_{Q_{L}} \times \mathrm{SU}(3)_{Q_{R}} \times$ $\mathrm{SU}(3)_{\ell_{L}} \times \mathrm{SU}(3)_{\ell_{R}}$ which is more economical and, unlike the SM, also simultaneously explains neutrino masses. ${ }^{1}$

We will therefore start with the gauge group $G_{L R} \equiv \mathrm{SU}(3)_{C} \times \mathrm{SU}(2)_{L} \times \mathrm{SU}(2)_{R} \times$ $\mathrm{U}(1)_{B-L} \times \mathrm{SU}(3)_{Q_{L}} \times \mathrm{SU}(3)_{Q_{R}} \times \mathrm{SU}(3)_{\ell_{L}} \times \mathrm{SU}(3)_{\ell_{R}}$, where $\mathrm{SU}(3)_{Q_{L}} \times \mathrm{SU}(3)_{Q_{R}}$ represents the flavor gauge symmetries respectively in the left- and right-handed quark sector, and $\mathrm{SU}(3)_{\ell_{L}} \times \mathrm{SU}(3)_{\ell_{R}}$ the corresponding ones for the lepton sector. The particle content and its transformation properties under fundamental representations of the group $G_{L R}$ are reported in table 1 . One can clearly note the one-to-one correspondence between the quark and the lepton multiplets, differing only in the behavior under $\mathrm{SU}(3)_{c}$. It is easy to verify that this field content makes $G_{L R}$ completely anomaly-free, separately in the quark and lepton sectors.

We next discuss the quark Yukawa couplings. We will ignore for the moment the leptonic ones since they do not have any effect on the final results for the quark sector. (The leptonic flavor symmetries are discussed in section 4.) In writing the quark Lagrangian at the $\mathrm{TeV}$ scale, we will generally assume that the gauge symmetry $\mathrm{SU}(2)_{L} \times \mathrm{SU}(2)_{R} \times$ $\mathrm{U}(1)_{B-L}$ is restored at that scale. Even under this assumption, one has still to specify where the parity symmetry is broken. As anticipated in the Introduction, one can either suppose that parity is restored at the $\mathrm{TeV}$ scale, or else that its restoration takes place at some much higher scale $M_{P}$ [21]. Let us first focus on the former case, namely of TeV-scale parity. In this case, the Lagrangian for the quark sector reads

$$
\begin{aligned}
\mathcal{L}_{\mathrm{q}}= & \mathcal{L}_{\mathrm{q}}^{\mathrm{kin}}-V\left(Y_{u}, Y_{d}, \chi_{L}, \chi_{R}\right)+\lambda_{u}\left(\bar{Q}_{L} \tilde{\chi}_{L} \psi_{R}^{u}+\bar{Q}_{R} \tilde{\chi}_{R} \psi_{L}^{u}\right)+\lambda_{d}\left(\bar{Q}_{L} \chi_{L} \psi_{R}^{d}+\bar{Q}_{R} \chi_{R} \psi_{L}^{d}\right) \\
& +\lambda_{u}^{\prime} \bar{\psi}_{L}^{u} Y_{u} \psi_{R}^{u}+\lambda_{d}^{\prime} \bar{\psi}_{L}^{d} Y_{d} \psi_{R}^{d}+\text { h.c. },
\end{aligned}
$$

where we have written explicitly only the Yukawa interactions. We note at this point that, since under parity $Q_{L} \leftrightarrow Q_{R}$ and $\psi_{L}^{u} \leftrightarrow \psi_{R}^{u}$ (and similarly for $\psi_{L, R}^{d}$ ), parity symmetry

\footnotetext{
${ }^{1}$ For other horizontal symmetry extensions of left-right models, see [22-24]. Furthermore, gauged flavor symmetries have also been discussed in the context of the Pati-Salam GUT in ref. [25], see appendix therein.
} 


\begin{tabular}{|c|c|c|c|c|c|c|c|c|}
\hline & $\mathrm{SU}(2)_{L}$ & $\mathrm{SU}(2)_{R}$ & $\mathrm{U}(1)_{B-L}$ & $\mathrm{SU}(3)_{Q_{L}}$ & $\mathrm{SU}(3)_{Q_{R}}$ & $\mathrm{SU}(3)_{c}$ & $\mathrm{SU}(3)_{\ell_{L}}$ & $\mathrm{SU}(3)_{\ell_{R}}$ \\
\hline$Q_{L}$ & 2 & & $\frac{1}{3}$ & 3 & & 3 & & \\
\hline$Q_{R}$ & & 2 & $\frac{1}{3}$ & & 3 & 3 & & \\
\hline$\psi_{L}^{u}$ & & & $\frac{4}{3}$ & & 3 & 3 & & \\
\hline$\psi_{R}^{u}$ & & & $\frac{4}{3}$ & 3 & & 3 & & \\
\hline$\psi_{L}^{d}$ & & & $-\frac{2}{3}$ & & 3 & 3 & & \\
\hline$\psi_{R}^{d}$ & & & $-\frac{2}{3}$ & 3 & & 3 & & \\
\hline$L_{L}$ & 2 & & -1 & & & & 3 & \\
\hline$L_{R}$ & & 2 & -1 & & & & & 3 \\
\hline$\psi_{L}^{e}$ & & & -2 & & & & & 3 \\
\hline$\psi_{R}^{e}$ & & & -2 & & & & 3 & \\
\hline$\psi_{L}^{\nu}$ & & & 0 & & & & & 3 \\
\hline$\psi_{R}^{\nu}$ & & & 0 & & & & 3 & \\
\hline$\chi_{L}$ & 2 & & 1 & & & & & \\
\hline$\chi_{R}$ & & 2 & 1 & & & & & \\
\hline$Y_{u}$ & & & & $\overline{3}$ & 3 & & & \\
\hline$Y_{d}$ & & & & $\overline{3}$ & 3 & & & \\
\hline$Y_{\ell}$ & & & & & & & $\overline{3}$ & 3 \\
\hline$Y_{\nu}$ & & & & & & & $\overline{3}$ & 3 \\
\hline
\end{tabular}

Table 1. Model content. For ease of readability, horizontal lines separate the quark multiplets, the lepton ones and the Higgs and flavon ones from each other, and only non-singlet transformation properties are reported explicitly.

requires $Y_{u, d} \leftrightarrow Y_{u, d}^{\dagger}$ and the $\lambda_{u, d}$ as well as $\lambda_{u, d}^{\prime}$ couplings to be real. ${ }^{2}$ Parity will thus be broken only by the different vevs of $\chi_{L, R}$ (the tilde on these fields in eq. (2.1) indicates $\tilde{\chi}=\tau_{2} \chi^{*}$ for both $\mathrm{L}$ and $\mathrm{R}$ ).

In the case where parity is broken at a scale $M_{P}$ much higher than the $\mathrm{TeV}$ [21], the interactions in eq. (2.1), obtained from each other by the parity operation defined in the previous paragraph, have in principle different couplings. For example

$$
\lambda_{u}\left(\bar{Q}_{L} \tilde{\chi}_{L} \psi_{R}^{u}+\bar{Q}_{R} \tilde{\chi}_{R} \psi_{L}^{u}\right) \rightarrow \lambda_{u L} \bar{Q}_{L} \tilde{\chi}_{L} \psi_{R}^{u}+\lambda_{u R} \bar{Q}_{R} \tilde{\chi}_{R} \psi_{L}^{u},
$$

because of different RGE running beneath the scale $M_{P}$. Hence, similarly as in eq. (2.2), in the case of no $\mathrm{TeV}$-scale parity we will distinguish the left and right instances of each gauge, $\lambda$ and $\lambda^{\prime}$ couplings by an $\mathrm{L}$ or $\mathrm{R}$ subscript.

Concerning the breaking of the gauge groups, the flavor gauge group $\mathrm{SU}(3)_{Q_{L}} \times$ $\mathrm{SU}(3)_{Q_{R}}$ is broken spontaneously by the vevs of $Y_{u}$ and $Y_{d}$ while the group $\mathrm{SU}(2)_{L} \times \mathrm{SU}(2)_{R}$ by the vevs of the Higgs doublets, $\chi_{L, R}$, as already mentioned. In particular, we adopt the

\footnotetext{
${ }^{2}$ In particular, concerning $\lambda_{u, d}$, one can note that there is one single such coupling for either of the up-type or down-type quark interactions with heavy fermions. Hence, one can remove possible phases in $\lambda_{u, d}$ by absorbing them in the $\psi^{u}$ and $\psi^{d}$ fields, respectively.
} 
following vev normalization

$$
\left\langle\chi_{L}\right\rangle=\left(\begin{array}{c}
0 \\
v_{L}
\end{array}\right), \quad\left\langle\chi_{R}\right\rangle=\left(\begin{array}{c}
0 \\
v_{R}
\end{array}\right),
$$

while diagonal $Y$ vevs will be denoted henceforth as $\left\langle\hat{Y}_{u, d}\right\rangle$.

Fermion masses. From eq. (2.1) one can read off the up-type fermion mass Lagrangian to be $\mathcal{L}_{m}=\overline{\mathcal{U}}_{L} M_{u} \mathcal{U}_{R}$, with $\mathcal{U}=\operatorname{column}\left\{u, \psi^{u}\right\}$, each of the $u$ and $\psi^{u}$ fields carrying a generation index. The mass matrix reads

$$
M_{u}=\left(\begin{array}{cc}
0 & \lambda_{u} v_{L} \mathbb{1}_{3 \times 3} \\
\lambda_{u} v_{R} \mathbb{1}_{3 \times 3} & \lambda_{u}^{\prime}\left\langle\hat{Y}_{u}\right\rangle
\end{array}\right), \quad M_{d}=\left(\begin{array}{cc}
0 & \lambda_{d} v_{L} \mathbb{1}_{3 \times 3} \\
\lambda_{d} v_{R} \mathbb{1}_{3 \times 3} & \lambda_{d}^{\prime}\left\langle\hat{Y}_{d}\right\rangle
\end{array}\right) .
$$

For the time being, we assume the parameters $\lambda_{u} v_{L}$ and $\lambda_{u} v_{R}$ to be much smaller than any of the $\lambda_{u}^{\prime}\left\langle\hat{Y}_{u}\right\rangle_{i}$. (With the subscript $i$ in $\left\langle\hat{Y}_{u(d)}\right\rangle_{i}$ we shall henceforth label the diagonal entries of the flavon vev matrices.) Then, to leading order in an expansion in the parameters $\frac{\lambda_{u} v_{L(R)}}{\lambda_{u}^{\prime}}\left\langle\hat{Y}_{u}\right\rangle_{i}^{-1}$, and analogous for the down sector, the above mass matrices assume the following diagonal form

$$
\hat{M}_{u} \simeq\left(\begin{array}{cc}
\frac{\lambda_{u}^{2} v_{L} v_{R}}{\lambda_{u}^{\prime}\left\langle\hat{Y}_{u}\right\rangle} & 0 \\
0 & \lambda_{u}^{\prime}\left\langle\hat{Y}_{u}\right\rangle
\end{array}\right), \quad \hat{M}_{d} \simeq\left(\begin{array}{cc}
\frac{\lambda_{d}^{2} v_{L} v_{R}}{\lambda_{d}^{\prime}\left\langle\hat{Y}_{d}\right\rangle} & 0 \\
0 & \lambda_{d}^{\prime}\left\langle\hat{Y}_{d}\right\rangle
\end{array}\right)
$$

From eq. (2.5) it is evident that off-diagonalities in the light-quark Yukawa couplings are inherited from off-diagonalities in the flavon vevs $\left\langle Y_{u, d}\right\rangle$. We note first that, even below the $v_{L}$ scale, it is always possible to have one of the flavon vevs in diagonal form through an appropriate redefinition of the $\psi_{L, R}^{u}$ and $\psi_{L, R}^{d}$ basis (see eq. (2.1)). We choose $Y_{d}$ to be that particular flavon multiplet. This amounts to three parameters, fixed by the down-type quark masses. $Y_{u}$ will then be chosen to have a vev pattern of the form

$$
\left\langle Y_{u}\right\rangle=V_{R}^{\dagger}\left\langle\hat{Y}_{u}\right\rangle V_{L}, \quad\left\langle Y_{d}\right\rangle=\left\langle\hat{Y}_{d}\right\rangle,
$$

with $V_{L, R}$ unitary. Note that, as already mentioned, $V_{L}=V_{R}=V$ in eq. (2.6) follows from the $\left\langle Y_{u}\right\rangle$ vev pattern being hermitian and hence parity symmetric. This amounts to six real parameters and three phases. Two of these phases can be absorbed as relative phases of two up-type quark fields relative to the third one. This gives the six real parameters and one phase to fit up-type quark masses and the CKM matrix. ${ }^{3}$

In the basis of eq. (2.6), and again in the temporary approximation $v_{R} \ll\left\langle\hat{Y}_{u, d}\right\rangle$, the Yukawa couplings of the SM (defined from the interactions $\bar{u}_{L} y_{u} u_{R}$ and $\bar{d}_{L} y_{d} d_{R}$ ) read

$$
y_{u}=\frac{\lambda_{u}^{2} v_{R}}{\lambda_{u}^{\prime}} V_{L}^{\dagger}\left\langle\hat{Y}_{u}\right\rangle^{-1} V_{R}, \quad y_{d}=\frac{\lambda_{d}^{2} v_{R}}{\lambda_{d}^{\prime}}\left\langle\hat{Y}_{d}\right\rangle^{-1}
$$

\footnotetext{
${ }^{3}$ We also note incidentally that, from the point of view of our discussion, the $\lambda_{u, d}^{\prime}$ couplings can in principle be absorbed into the definition of the $Y_{u, d}$ vevs respectively. This effectively leaves as free parameters only $\lambda_{u, d}$, besides the scale of the vev $v_{R}$ and the $\mathrm{SU}(2)_{R}$ coupling $g_{R}$, making the model very economical.
} 
One can now rotate the $u_{L, R}$ fields as

$$
u_{L(R)}=V_{L(R)}^{\dagger} \hat{u}_{L(R)}, \quad d_{L(R)}=\hat{d}_{L(R)} .
$$

In the hatted basis, $y_{u}$ is diagonal and $V_{L}$ is moved to the $\bar{u}_{L} \gamma^{\mu} W_{\mu} d_{L}$ interaction. Therefore, $V_{L}$ can be interpreted as the CKM matrix, $V_{\mathrm{CKM}}$. As already stated, in our case of leftright symmetry we have strictly $V_{L}=V_{R}=V$ at the scale $v_{R}$. However, since we are interested only in $v_{R}$ values not very far from the electroweak symmetry breaking scale $v_{L}$, the radiative corrections to the above relation between left and right CKM's are expected to be small. Therefore, we will henceforth generally identify

$$
V_{L}=V_{R}=V_{\mathrm{CKM}},
$$

with caveats to be commented upon more below in the analysis. Since quark masses are given by $y_{i} v_{L}$, we can draw some conclusions from the approximate relations (2.7):

(i) In the limit of $v_{R} \ll\left\langle\hat{Y}_{u, d}\right\rangle_{i}$ the elements of the diagonal $\left\langle\hat{Y}_{u, d}\right\rangle$ matrices follow an inverted hierarchy with respect to the quark masses [10-13].

(ii) For a given value of $v_{R}$ and of the $\lambda^{(\prime)}$ couplings, eqs. (2.7) or the corresponding exact expressions in section 3.4 allow to univocally fix the $\left\langle\hat{Y}_{u, d}\right\rangle$ entries. Since the $Y_{u, d}$ vevs set also the mass scale for the flavor gauge bosons (see below in this section for details), the inverted hierarchy mentioned in item (i) implies a similar hierarchy in new flavor changing neutral current effects: the lighter the generations, the more suppressed the effects [10]. This is arguably one of the most attractive features of the model. We will return to this quantitatively in section 3.1.

(iii) In the exact parity case, the mass matrices $M_{u, d}$, see eq. (2.4), lead to $\arg \operatorname{det}\left[M_{u, d}\right]=0$, implying that the strong CP parameter at the tree level vanishes. The one loop calculation for a more general case of this type was carried out in ref. [20]. Using this result, we conclude that the model solves the strong $\mathrm{CP}$ problem without the axion.

Since the condition $v_{R} \ll\left\langle\hat{Y}_{u, d}\right\rangle_{i}$ may in general not hold for all flavors, we need to give for the quark masses a more exact relation than eq. (2.5). In fact, the fermion mixing matrices (2.4) can be diagonalized exactly. This will be discussed in section 3.4, along with its phenomenological consequences.

Flavor gauge boson masses. The masses of the $\mathrm{SU}(3)_{Q_{L}} \times \mathrm{SU}(3)_{Q_{R}}$ gauge bosons $G_{i L, R}(i=1, \ldots, 8)$ are obtained from the kinetic terms of $Y_{u}$ and $Y_{d}$ in the Lagrangian, $\operatorname{Tr}\left(\left|D^{\mu} Y_{u, d}\right|^{2}\right)$, where the covariant derivatives are

$$
D^{\mu} Y_{u, d}=\partial^{\mu} Y_{u, d}-i g_{H} G_{R}^{\mu} Y_{u, d}+i g_{H} Y_{u, d} G_{L}^{\mu} .
$$

The relevant mass terms read

$$
\begin{aligned}
\mathcal{L} & =\operatorname{Tr}\left(\left|g_{H} G_{R}^{\mu}\left\langle Y_{u}\right\rangle-g_{H}\left\langle Y_{u}\right\rangle G_{L}^{\mu}\right|^{2}\right)+\operatorname{Tr}\left(\left|g_{H} G_{R}^{\mu}\left\langle Y_{d}\right\rangle-g_{H}\left\langle Y_{d}\right\rangle G_{L}^{\mu}\right|^{2}\right) \\
& =\frac{1}{2} \mathcal{G}_{k}\left(M_{V}^{2}\right)_{k l} \mathcal{G}_{l},
\end{aligned}
$$


where $\mathcal{G}_{k} \equiv\left\{G_{L}^{a}, G_{R}^{a}\right\}$ is a vector containing the 16 fields in $G_{L, R}^{\mu}=G_{L, R}^{\mu a} \frac{\lambda^{a}}{2}$. The $\mathcal{G}_{k}$ are rotated by an orthogonal matrix $O$ such that $\mathcal{G}_{k}=O_{k j} \hat{\mathcal{G}}_{j}$, where $\hat{\mathcal{G}}_{i}$ are mass eigenstates.

One interesting point to note is that all the flavor gauge boson masses are determined by basically only one $Y$ vev, namely the largest of the $Y_{u}$ vevs $\left\langle\hat{Y}_{u}\right\rangle_{1}$. In fact, for the lightest among the $M_{\mathcal{G}_{i}},\left\langle\hat{Y}_{u}\right\rangle_{1}$ is multiplied by two powers of the Cabibbo angle $\theta_{C}$ (in the limit $\theta_{C} \rightarrow 0$, one gets at least one massless $\left.\mathcal{G}_{i}\right)$ and $\left\langle\hat{Y}_{u}\right\rangle_{1} \times \theta_{C}^{2}$ is larger than the second-largest $Y$ vev contribution, $\left\langle\hat{Y}_{u}\right\rangle_{2}$.

\section{Phenomenology}

In the subsections of this section we will discuss the various observables that are expected to provide a constraint (or else the possibility of a signal) for the model. Since in some cases - starting from the model spectrum - the model predictions vary in a wide range, we found it useful to explore these predictions with a flat scan of the model parameters. In the case where parity is assumed to be restored at the $\mathrm{TeV}$ scale, ranges have been chosen as follows:

1. $g_{H} \in[0.3,0.9]$ and $v_{R}$ such that $M_{W_{R}} \in[0.2,5] \mathrm{TeV}$ (the bound on $M_{W_{R}}$ from applicable constraints is taken into account afterwards).

2. $\lambda_{u} \in[0.96,5], \lambda_{d} \in[0.1,5]$, see discussion below eq. (3.9).

3. Setting the couplings $\lambda_{u, d}^{\prime}=1$, one effectively absorbs them into the definition of the $Y_{u, d}$ vevs, respectively (the relevant combination entering fermion mixing is $\lambda_{u, d}^{\prime} Y_{u, d}$, see eqs. (2.4)). This assumption is however restrictive for the mass spectrum of the flavor gauge bosons, that depends on $\left\langle\hat{Y}_{u, d}\right\rangle$, but not on $\lambda_{u, d}^{\prime}$, see eq. (2.11). Therefore, we have also scanned $\lambda_{u, d}^{\prime} \in[0.1,5]$.

4. Finally, we have taken $g_{L}=g_{R} \simeq 0.65$ for the $\mathrm{SU}(2)_{L, R}$ couplings.

In the other scenario where parity is not a good symmetry at the $\mathrm{TeV}$ scale, all the left vs. right couplings can be chosen as different from each other. Concerning the $\mathrm{SU}(2)_{L, R}$ couplings, in [21] examples have been given of scenarios where $g_{R} / g_{L} \sim 0.70$ for a UV complete theory which conserves parity. Here we therefore limit ourselves to the reference choice $g_{R}=0.7 \cdot g_{L}$, Concerning the other parameters:

- The left and right instances of the $g_{H}$ and $\lambda_{u, d}^{\prime}$ couplings have been scanned in the same ranges as specified in items 1 and 3 respectively.

- With regards to item 2 , we have scanned $\lambda_{u L} \in[0.96,5]$ and the rest of the $\lambda$ parameters in $[0.1,5]$.

- We have further enforced that the left vs. right instances of each coupling do not differ from each other by more than a factor of 5 . 
Two concluding comments concern the reality of the $\lambda^{(\prime)}$ couplings and the hermiticity of the Yukawa vevs, implicitly assumed in the above items. From the discussion below eq. (2.1), one can argue that, in the case where parity is not a good $\mathrm{TeV}$-scale symmetry, non-negligible complex phases may be present in (some of) the $\lambda^{(\prime)}$ couplings. This may in turn have an impact on $\mathrm{CP}$ violating observables, which are, however, not the main concern in this paper, for the reasons mentioned in section 3.2. Finally, departures from hermiticity in the Yukawa vevs correspond (see discussion beneath eq. (2.6)) to assuming sensible departures from eq. (2.9). Throughout this paper we neglect such effects. Again, we will comment on this assumption in section 3.2 .

\subsection{Tree-level FCNC effects}

The flavor gauge bosons $G_{L, R}^{\mu a}$ couple to the currents $\mathcal{J}_{H L, R}^{\mu a} \equiv g_{H} \bar{Q}_{L, R} \gamma^{\mu} \frac{\lambda^{a}}{2} Q_{L, R}$. Similarly as in ref. [10], these interactions give rise to new, tree-level, contributions to the 4 -fermion operators

$$
\begin{aligned}
Q_{1}^{q_{j} q_{i}} & =\left(\bar{q}_{i}^{\alpha} \gamma_{L}^{\mu} q_{j}^{\alpha}\right)\left(\bar{q}_{i}^{\beta} \gamma_{\mu, L} q_{j}^{\beta}\right), \\
\tilde{Q}_{1}^{q_{j} q_{i}} & =\left.Q_{1}^{q_{j} q_{i}}\right|_{L \rightarrow R}, \\
Q_{5}^{q_{j} q_{i}} & =\left(\bar{q}_{i}^{\alpha} P_{L} q_{j}^{\beta}\right)\left(\bar{q}_{i}^{\beta} P_{R} q_{j}^{\alpha}\right),
\end{aligned}
$$

with Latin and Greek indices on the quark fields denoting flavor and respectively color, and where $P_{L, R} \equiv\left(1 \mp \gamma_{5}\right) / 2$. In the quark mass eigenstates basis, the Wilson coefficients of the above operators read

$$
\begin{aligned}
C_{1}^{q_{j} q_{i}} & =-\frac{g_{H}^{2}}{8}\left(M_{V}^{2}\right)_{a, b}^{-1}\left(V_{L}^{q} \lambda^{a} V_{L}^{q \dagger}\right)_{i j}\left(V_{L}^{q} \lambda^{b} V_{L}^{q \dagger}\right)_{i j}, \\
\tilde{C}_{1}^{q_{j} q_{i}} & =-\frac{g_{H}^{2}}{8}\left(M_{V}^{2}\right)_{8+a, 8+b}^{-1}\left(V_{R}^{q} \lambda^{a} V_{R}^{q \dagger}\right)_{i j}\left(V_{R}^{q} \lambda^{b} V_{R}^{q \dagger}\right)_{i j}, \\
C_{5}^{q_{j} q_{i}} & =\frac{g_{H}^{2}}{2}\left(M_{V}^{2}\right)_{a, 8+b}^{-1}\left(V_{L}^{q} \lambda^{a} V_{L}^{q \dagger}\right)_{i j}\left(V_{R}^{q} \lambda^{b} V_{R}^{q \dagger}\right)_{i j},
\end{aligned}
$$

where $q$ can be $u$ or $d$, and a sum over $a$ and $b$ in the range $1, \ldots, 8$ is understood. The matrices $V_{L, R}^{u, d}$ rotating the $u, d$ fields from the flavor to the mass eigenbasis should be chosen as

$$
V_{L, R}^{u}=V_{L, R}, \quad V_{L, R}^{d}=\mathbb{1},
$$

compatibly with eq. (2.8) and in the approximation of neglecting the mixing between quarks and heavy fermion states.

Updated bounds on the Wilson coefficients in eq. (3.2) have been reported by the UTfit collaboration [26] and usefully tabulated in their table 4 for the different meson-antimeson mixing processes. The contributions, predicted in our model, to the above coefficients have been explored by the random scan mentioned at the beginning of section 3. As previously anticipated, these contributions are well within the existing bounds in the bulk of the explored parameter space. As an illustration, we report in figure 1 the largest in magnitude among these contributions, that to the $K-\bar{K}$ mixing coefficient $\operatorname{Re}\left(C_{K}^{5}\right)$, in the case of $\mathrm{TeV}$-scale parity. The $M_{W_{R}}$ mass is therefore mostly bounded from box diagrams 


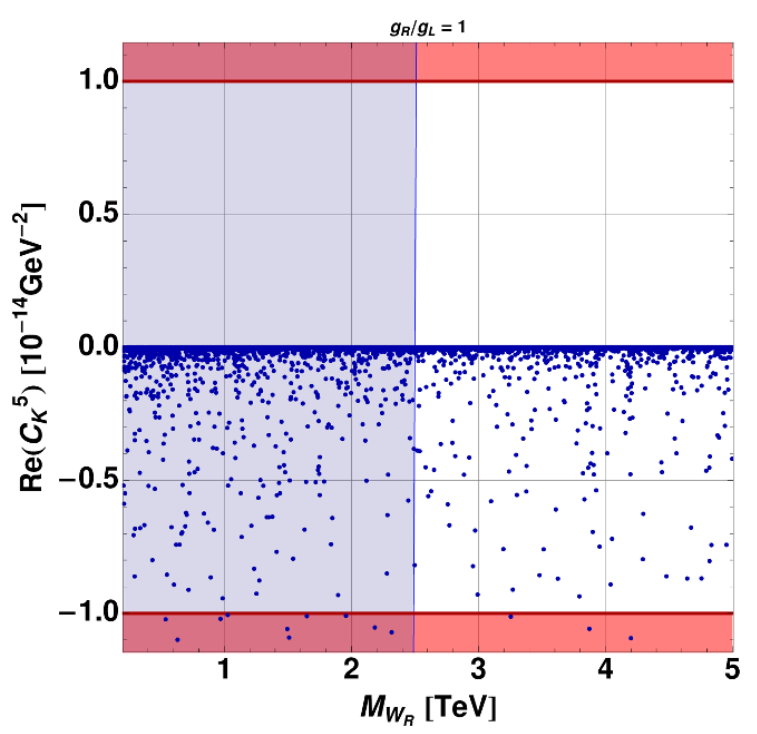

Figure 1. $M_{W_{R}}$ vs. the contribution to the $\Delta F=2$ Wilson coefficient $\operatorname{Re}\left(C_{K}^{5}\right)$. The horizontal (red) shaded regions are excluded from the analysis of ref. [26]. The vertical (blue) region extending leftwards is the $M_{W_{R}}$ bound from loop FCNCs [27-31].
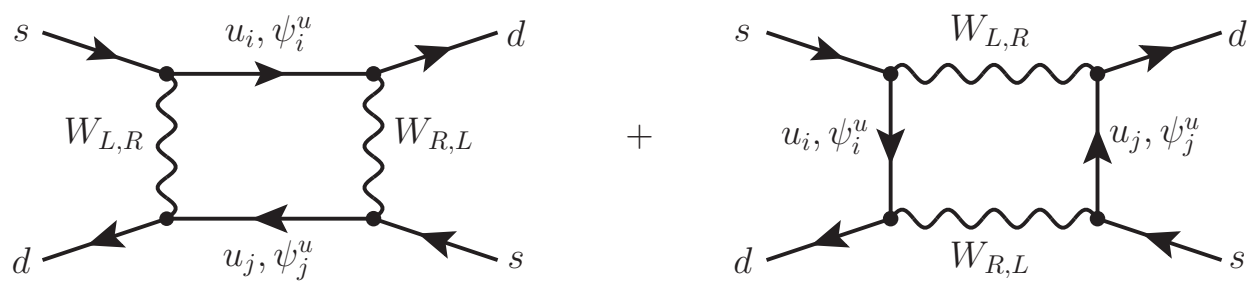

Figure 2. Box diagrams contributing to the $K_{L}-K_{S}$ mass difference in our model.

with $W_{R}$ exchange, as discussed in section 3.2 . The resulting bound, $M_{W_{R}} \gtrsim 2.5 \mathrm{TeV}[27-$ 31], is shown in figure 1 as a vertical shaded area extending leftwards. On the $M_{G_{i}}$ masses, on the other hand, we will return in section 3.3.

\subsection{Loop FCNC effects and lower bound on the $W_{R}$ mass}

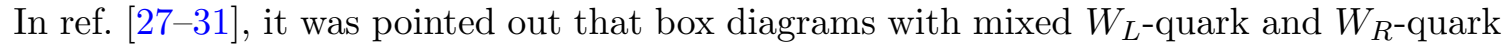
exchange provide a severe lower bound on the $\mathrm{SU}(2)_{R}$-symmetry breaking scale in the case of the minimal LR symmetric model with Higgs bidoublets. This happens on account of the constraint from the $K_{L}-K_{S}$ mass difference, $\Delta M_{K}$, and the bound reads $M_{W_{R}} \gtrsim 2.5 \mathrm{TeV}{ }^{4}$

In our model, beside the known quarks, also their heavy fermionic partners propagate in the box diagrams, because of fermion mixing. The full amplitude, for a given quarkheavy fermion doublet (e.g. $t$ and $\psi_{u, 3}$ ), is depicted in figure 2 . The sum of the contributions turns out to display a GIM-like mechanism of cancellation, as we will shortly explain.

\footnotetext{
${ }^{4}$ Even more stringent bounds would come from $\mathrm{CP}$ violating observables again in the $K$ sector, but these bounds are much more model dependent [32].
} 
First note that the upper fermion line in any of the box diagrams of figure 2 will be proportional to a factor of $V_{R}^{\dagger} \cos \left(\theta_{i, R}^{u}\right) m_{i}^{u} \cos \left(\theta_{i, L}^{u}\right) V_{L}$ or $V_{R}^{\dagger} \sin \left(\theta_{i, R}^{u}\right) M_{i}^{u} \sin \left(\theta_{i, L}^{u}\right) V_{L}$ depending on whether the fermion is a quark or its heavy partner (in the lower fermion lines one will have the hermitian conjugate of the same expressions). Assuming propagation of a single quark species, it is therefore easy to see that $\Delta M_{K}$ is such that

$$
\Delta M_{K} \propto g_{L}^{2} g_{R}^{2}\left|V_{R}^{\dagger} c_{i_{R}} c_{i_{L}}\left(\frac{m_{i}}{p^{2}-m_{i}^{2}}-\frac{t_{i_{R}} M_{i} t_{i_{L}}}{p^{2}-M_{i}^{2}}\right) V_{L}\right|_{12}^{2},
$$

where we have abbreviated $\cos , \tan$ with $c, t$ and dropped the superscript on the masses. One should note at this point that

a. the mass eigenvalues for quarks and heavy fermions come with opposite signs (cf. eq. (2.4)),

b. $m_{i}$ and $t_{i_{R}} M_{i} t_{i_{L}}$ are equal, as can be seen by using eqs. (3.8), namely that the generally small mixing angles are compensated by a large mass in the second term in the parenthesis of eq. (3.4),

c. after factoring out the common mass term mentioned in item b, the diagram is proportional to $m_{i}^{2}-M_{i}^{2}$, as in the GIM mechanism. In our model the mass difference between a quark and its fermionic partner is smallest in the top sector. Interestingly, in most of the meson mixings' phenomenology of the down-sector, including the CP violating observable $\epsilon_{K}$, the top contribution is the most important one.

The observation in item $\mathrm{c}$ has the potential of substantially weakening the severe bounds on the parity breaking scale coming from $\epsilon_{K}$ [27-31], and we reserve to come back to this issue in a separate study. As stated elsewhere, the predictions for $\mathrm{CP}$ violating observables are however quite model-dependent, and here we confine our discussion to $\mathrm{CP}$ conserving ones, in particular $\Delta M_{K}$. In this case, the dominant loop contribution comes from the charm sector, hence the mechanism described above is much less effective, since the quark - heavy fermion splitting is very large. On account of this constraint, we find that the lower bound on the $W_{R}$ mass from $K_{L}-K_{S}$ mass difference coincides with that in ref. [27-31], namely we get $M_{W_{R}} \gtrsim 2.5 \mathrm{TeV}$. This bound holds in the exact parity case $\left(g_{R} / g_{L}=1\right)$, that we have been assuming in this discussion.

On the other hand, if parity is broken at a scale much higher than the TeV scale, one expects a splitting in the $\mathrm{TeV}$-scale values of $g_{L}$ and $g_{R}$ in eq. (3.4), and values of $\left(g_{R} / g_{L}\right)^{2}<1$ provide a further suppression of eq. (3.4) by the same factor. For example, assuming $g_{R} / g_{L}=0.7$, this bound scales down to $M_{W_{R}} \gtrsim 1.7 \mathrm{TeV}$. One can see this by simply noting that, as far as $M_{W_{R}}$ and the $\mathrm{SU}(2)_{L, R}$ gauge couplings are concerned, $\Delta M_{K}$ scales as $\Delta M_{K} \propto g_{L}^{2} g_{R}^{2} / M_{W_{R}}^{2}$, and that the $\Delta M_{K}$ calculation in the SM is dominated by loops mediated by the charm quark, whose vectorlike partner $\psi_{2}^{u}$ is, to first approximation, decoupled. As discussed at the beginning of section 3 the choice $g_{R} / g_{L}=0.7$ [21] will be our reference one for the scenario of no $\mathrm{TeV}$-scale parity.

Two further comments are in order here. First, we note that a choice such as $g_{R} / g_{L}=$ 0.7 will also affect the $W_{R}$ collider bound since the $W_{R}$ production rate will go down by the 


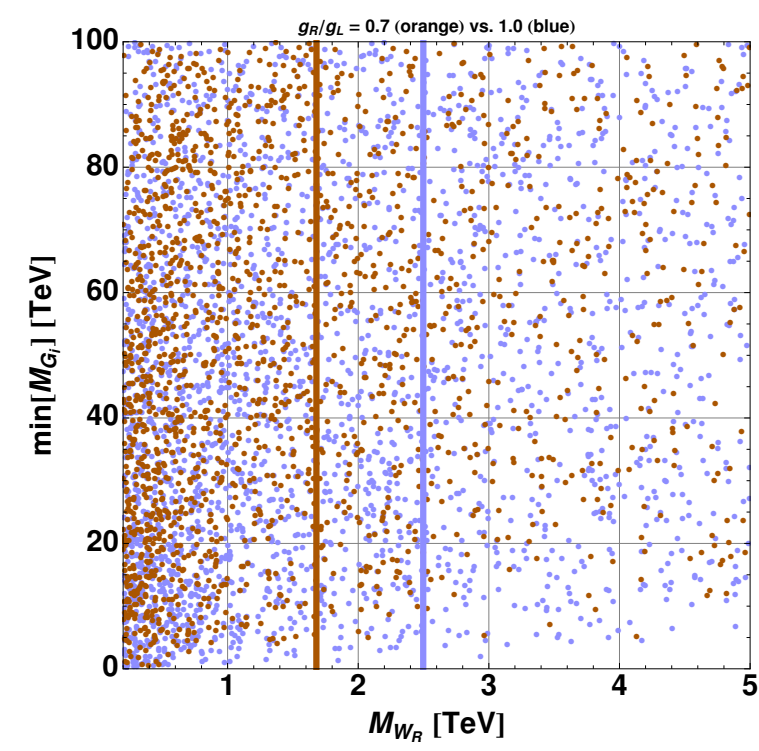

Figure 3. $M_{W_{R}}$ vs. the mass of the lightest flavor gauge boson, $\min \left(M_{\mathcal{G}_{i}}\right)$, for $g_{R} / g_{L}=0.7$ (orange dots) or 1.0 (blue dots).

factor $\left(g_{R} / g_{L}\right)^{2}$ as well. Second, high-scale parity breaking will in general also cause some misalignment of the left and right CKM matrices. In particular, large off-diagonal entries in the right CKM matrix have the potential of correspondingly increasing the contributions to flavor observables, a simple example being, again, that of meson anti-meson mixings. The interest of this example is in the fact that the potential phenomenology of these contributions encompasses not only flavor violation, but also mixing-induced $\mathrm{CP}$ violation, namely observables like $\left|\epsilon_{K}\right|, \sin 2 \beta$ and $\sin 2 \beta_{s}{ }^{5}$

For the aims of the present discussion, we will assume that CKM entries undergo corrections due to RGE that don't modify their hierarchical structure, hence that the induced misalignment between $V_{L}$ and $V_{R}$ is small enough not to grossly alter the main argument of this section. A more detailed answer can be given, we feel, only in the context of specific models.

\subsection{Flavor gauge boson mass scale}

As mentioned above, from the point of view of flavor violating effects mediated by $\mathcal{G}_{i}$ exchange, the model is compatible with $M_{\mathcal{G}_{i}}$ as small as $\mathrm{O}(\mathrm{TeV})$, and this represents a potentially interesting new signal. Of course the question arises here, whether there are other model constraints placing a more stringent lower bound on this mass. From eq. (2.11), one expects the $\mathcal{G}_{i}$ spectrum to be correlated with the Yukawa vevs, which are fixed, in the combinations $\lambda_{u}^{\prime}\left\langle Y_{u}\right\rangle$ and $\lambda_{d}^{\prime}\left\langle Y_{d}\right\rangle$, by the requirement that, after fermion mixing, quark masses have the observed values. Therefore, with fixed $\lambda^{\prime}\langle Y\rangle$ 's, one may expect lower

\footnotetext{
${ }^{5}$ On the other hand, we do not expect large effects to these observables to come from the other potential sources of flavor mixing discussed in this paper, namely tree-level FCNCs mediated by flavor gauge bosons (see section 3.1) or quark — heavy-fermion mixing (see section 3.4.2).
} 
$\mathcal{G}_{i}$ masses to occur for larger $\lambda^{\prime}$ couplings. This $M_{\mathcal{G}_{i}}$ vs. $\lambda_{u, d}^{\prime}$ correlation is, however, completely smeared out by the freedom in the choice of the coupling $g_{H}$.

A correlation (albeit again not very sharp) is instead observed between the lowest allowed $\mathcal{G}_{i}$ mass and the $\mathrm{SU}(2)_{R}$-breaking scale $v_{R}$, which, in turn, is related to the $M_{W_{R}}$ bound discussed in section 3.2. This correlation, and the applicable $M_{W_{R}}$ bound, is shown in figure 3 in the two cases of exact TeV-scale parity (meaning $g_{R} / g_{L}=1$ ) and of no $\mathrm{TeV}$-scale parity (where we assume, as mentioned $g_{R} / g_{L}=0.7[21]$ ). From the lowermost points, one can see that the minimum $M_{\mathcal{G}_{i}}$ mass tends to grow with growing $M_{W_{R}}$. In particular, the lowermost red (blue) points on the right of the red (blue) vertical line imply allowed $M_{\mathcal{G}_{i}}$ values going down to about $3 \mathrm{TeV}$.

\subsection{Fermion mixing and its consequences}

The fermion mixing matrix $M_{u}$ in eq. (2.4) can be diagonalized via orthogonal transformations acting separately on each generation $i$ and each chirality, namely:

$$
\left(\begin{array}{c}
\hat{u}_{i} \\
\hat{\psi}_{i}^{u}
\end{array}\right)_{L(R)}=\mathcal{R}\left(\theta_{L(R), i}^{u}\right)\left(\begin{array}{c}
u_{i} \\
\psi_{i}^{u}
\end{array}\right)_{L(R)}, \quad \text { with } \mathcal{R}(\theta) \equiv\left(\begin{array}{cc}
\cos \theta-\sin \theta \\
\sin \theta & \cos \theta
\end{array}\right),
$$

where the hats denote mass eigenstates. This eingenvalue problem admits the following analytic solution (we recall again that the diagonal entries of the flavon vev matrices are denoted with $\left.\left\langle\hat{Y}_{u(d)}\right\rangle_{i}\right)$

$$
\begin{aligned}
\left(m_{u, i}^{ \pm}\right)^{2} & =\frac{1}{2}\left(\left(\lambda_{u}^{\prime}\left\langle\hat{Y}_{u}\right\rangle_{i}\right)^{2}+\lambda_{u}^{2}\left(v_{L}^{2}+v_{R}^{2}\right) \pm \Delta_{i}^{u}\right), \\
\text { with } \quad \Delta_{i}^{u} & \equiv \sqrt{\left(\lambda_{u}^{\prime}\left\langle\hat{Y}_{u}\right\rangle_{i}\right)^{4}+2\left(\lambda_{u}^{\prime}\left\langle\hat{Y}_{u}\right\rangle_{i}\right)^{2} \lambda_{u}^{2}\left(v_{L}^{2}+v_{R}^{2}\right)+\lambda_{u}^{4}\left(v_{L}^{2}-v_{R}^{2}\right)^{2}}
\end{aligned}
$$

with rotation angles given by

$$
\begin{aligned}
\tan \theta_{L, i}^{u} & =\frac{\lambda_{u}^{2}\left(v_{L}^{2}-v_{R}^{2}\right)-\left(\lambda_{u}^{\prime}\left\langle\hat{Y}_{u}\right\rangle_{i}\right)^{2}+\Delta_{i}^{u}}{2 \lambda_{u} v_{L} \lambda_{u}^{\prime}\left\langle\hat{Y}_{u}\right\rangle_{i}} \\
\tan \theta_{R, i}^{u} & =\frac{\lambda_{u}^{2}\left(v_{R}^{2}-v_{L}^{2}\right)-\left(\lambda_{u}^{\prime}\left\langle\hat{Y}_{u}\right\rangle_{i}\right)^{2}+\Delta_{i}^{u}}{2 \lambda_{u} v_{R} \lambda_{u}^{\prime}\left\langle\hat{Y}_{u}\right\rangle_{i}} .
\end{aligned}
$$

Provided the $\lambda, \lambda^{\prime}$ couplings are of the same order, we will generally have $\left(\lambda_{u} v_{L}\right)^{2} \ll$ $\left(\lambda_{u} v_{R}\right)^{2},\left(\lambda_{u}^{\prime}\left\langle\hat{Y}_{u}\right\rangle_{i}\right)^{2}$. Accordingly expanding the above formulae one obtains the following approximate, but accurate solution $\left(x_{i} \equiv\left(\lambda_{u} v_{L} / \lambda_{u}^{\prime}\left\langle\hat{Y}_{u}\right\rangle_{i}\right)^{2}\right)$

$$
\begin{aligned}
\left(m_{u, i}^{-}\right)^{2} & =\frac{\lambda_{u}^{4} v_{L}^{2} v_{R}^{2}}{\lambda_{u}^{2} v_{R}^{2}+\left(\lambda_{u}^{\prime}\left\langle\hat{Y}_{u}\right\rangle_{i}\right)^{2}}+\mathrm{O}\left(x_{i}^{2}\right), & \left(m_{u, i}^{+}\right)^{2}=\lambda_{u}^{2} v_{R}^{2}+\left(\lambda_{u}^{\prime}\left\langle\hat{Y}_{u}\right\rangle_{i}\right)^{2}+\mathrm{O}\left(x_{i}\right), \\
\tan \theta_{L, i}^{u}= & =\frac{\lambda_{u} v_{L} \lambda_{u}^{\prime}\left\langle\hat{Y}_{u}\right\rangle_{i}}{\lambda_{u}^{2} v_{R}^{2}+\left(\lambda_{u}^{\prime}\left\langle\hat{Y}_{u}\right\rangle_{i}\right)^{2}}+\mathrm{O}\left(x_{i}^{3 / 2}\right), & \tan \theta_{R, i}^{u}=\frac{\lambda_{u} v_{R}}{\lambda_{u}^{\prime}\left\langle\hat{Y}_{u}\right\rangle_{i}}+\mathrm{O}\left(x_{i}\right) .
\end{aligned}
$$

Here $m^{-}$and $m^{+}$are to be identified respectively with the quark and heavy partners masses. A completely analogous solution exists in the down sector and is obtained from the above formulae with just the substitution $u \rightarrow d$. Note that the quark masses implied 
by the approximate $y_{u, d}$ in eq. (2.7) can be obtained from the $m_{u, i}^{-}$in eq. (3.8) in the limit $\lambda_{u} v_{R} \ll \lambda_{u}^{\prime}\left\langle\hat{Y}_{u}\right\rangle_{i}$

Let us focus on the general solution in eqs. (3.6) and (3.7). For fixed $\lambda_{u}$ and $v_{R}$, one can determine the combination $\lambda_{u}^{\prime}\left\langle\hat{Y}_{u}\right\rangle_{i}$ by inverting the equations $m_{u, i=1,2,3}^{-}=m_{u, c, t}$, with the up-type quark mass values on the r.h.s. These equations admit a real solution in $\lambda_{u}^{\prime}\left\langle\hat{Y}_{u}\right\rangle_{i}$ only for sufficiently high $\lambda_{u}$. Note in fact that, with fixed values for the other parameters, the maximum of $\left(m_{u, i}^{-}\right)^{2}$ occurs for $\lambda_{u}^{\prime}\left\langle\hat{Y}_{u}\right\rangle_{i}=0$ (see also the first of eqs. (3.8)). Necessary condition for a real solution to exist is therefore

$$
m_{u, c, t}<\left.m_{u, i}^{-}\left(\lambda_{u}^{\prime}\left\langle\hat{Y}_{u}\right\rangle_{i}=0\right)\right|_{i=1,2,3}=\lambda_{u} v_{L},
$$

where we have assumed $v_{L}<v_{R}$. Since $v_{L} \simeq 174 \mathrm{GeV}$ - very close to $m_{t}$ - the above inequality implies $\lambda_{u} \gtrsim 0.94$. (From the analogous inequalities in the down-quark sector, one also derives $\lambda_{d} \gtrsim 0.02$.) Note as well that, for the boundary value $\lambda_{u}=0.94$, the solution of eq. (3.9) for $i=3$ is $\lambda_{u}^{\prime}\left\langle\hat{Y}_{u}\right\rangle_{3}=0$. This explains the lower bound chosen for $\lambda_{u}$ in our scans, see beginning of section 3 .

Parity-broken case. In the case where parity is not a good symmetry at the TeV scale, we would expect the light quark Yukawa couplings to be left-right asymmetric as already noted. In this case, the formulae for quark as well as heavy-fermion masses and mixings change accordingly, i.e. eqs. (3.8) are replaced by

$$
\begin{aligned}
& \left(m_{u, i}^{-}\right)^{2}=\frac{\lambda_{u L}^{2} \lambda_{u R}^{2} v_{L}^{2} v_{R}^{2}}{\lambda_{u R}^{2} v_{R}^{2}+\left(\lambda_{u}^{\prime}\left\langle\hat{Y}_{u}\right\rangle_{i}\right)^{2}}+\mathrm{O}\left(x_{i}^{2}\right), \quad\left(m_{u, i}^{+}\right)^{2}=\lambda_{u R}^{2} v_{R}^{2}+\left(\lambda_{u}^{\prime}\left\langle\hat{Y}_{u}\right\rangle_{i}\right)^{2}+\mathrm{O}\left(x_{i}\right), \\
& \tan \theta_{L, i}^{u}=\frac{\lambda_{u L} v_{L} \lambda_{u}^{\prime}\left\langle\hat{Y}_{u}\right\rangle_{i}}{\lambda_{u R}^{2} v_{R}^{2}+\left(\lambda_{u}^{\prime}\left\langle\hat{Y}_{u}\right\rangle_{i}\right)^{2}}+\mathrm{O}\left(x_{i}^{3 / 2}\right), \quad \tan \theta_{R, i}^{u}=\frac{\lambda_{u R} v_{R}}{\lambda_{u}^{\prime}\left\langle\hat{Y}_{u}\right\rangle_{i}}+\mathrm{O}\left(x_{i}\right) .
\end{aligned}
$$

This case becomes very similar to the GRV examples [10] and as we see from the figures below allows vectorlike quark masses of about $2 \mathrm{TeV}$, making them, in principle, accessible at the LHC. If $\lambda_{u(d) R} \ll \lambda_{u(d) L}$, then the vectorlike quark masses could be even lighter, as is clear from eq. (3.10).

The one difference from the $\mathrm{TeV}$-scale parity case is that there is no reason for $\lambda_{u, d}^{\prime}$ to be real and therefore the model does not solve the strong CP problem. However, if there is parity restoration at some high scale, at that scale one does have a solution to the strong $\mathrm{CP}$ problem and an extrapolation is necessary to estimate how large a $\theta$ is induced at low energy. This kind of analysis is beyond the scope of this paper and we hope to take it up separately.

Bounds on heavy-fermion masses and mixings. The interesting phenomenological question is that of the magnitude of the mixing angles and of the lowest allowed masses $M_{\psi_{i}^{u}}$ and $M_{\psi_{i}^{d}}$ for the heavy up-type and down-type fermion partners. Note that these masses are given by the $m_{u, i}^{+}$and $m_{d, i}^{+}$solutions in eq. (3.8). According to these equations, the heavy fermion masses are correlated with both the $\mathrm{SU}(2)_{R}$-breaking scale $v_{R}$ and with the scales of gauge flavor symmetry breaking. In practice the latter correlation is blurred by the dependence on the unknown $\lambda$ and $\lambda^{\prime}$ parameters. On the other hand, the correlation with 


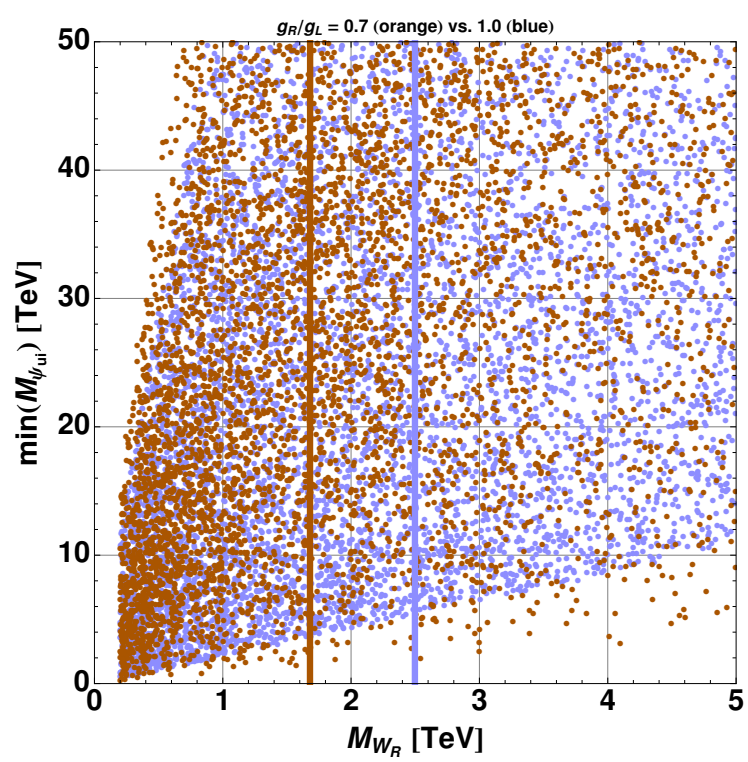

Figure 4. $M_{W_{R}}$ vs. the mass of the lightest (up-type) fermionic partner, for $g_{R} / g_{L}=0.7$ (orange dots) or 1.0 (blue dots). The vertical lines - again in orange or blue for $g_{R} / g_{L}=0.7$ or 1 represent the $M_{W_{R}}$ bound discussed in section 3.2.

$v_{R}$ still allows to infer the lowest allowed values for $M_{\psi_{i}^{u}}$ and $M_{\psi_{i}^{d}}$, taking into account the $M_{W_{R}}$ bound discussed in section 3.2. The situation is illustrated in figure 4, that displays the lightest up-type fermion partner mass vs. $M_{W_{R}}$. Blue and respectively orange dots refer to the parity vs. no-parity scans, see beginning of section 3 for details. The vertical lines represent the corresponding $M_{W_{R}}$ bounds. One can see that, while the exact parity case seems to exclude $M_{\psi_{i}^{u}} \lesssim 5 \mathrm{TeV}$, in the no parity case masses going down to $2 \mathrm{TeV}$ or even lower are possible. We mention that we found similar values to be possible also for the lightest down-type heavy fermion masses. In fact, as evident already from the first of eqs. (3.8), a large $m_{t} / m_{b}$ ratio does not necessarily imply a corresponding hierarchy in $\lambda_{d}^{\prime}\left\langle\hat{Y}_{d}\right\rangle_{3} / \lambda_{u}^{\prime}\left\langle\hat{Y}_{u}\right\rangle_{3}$ because $\lambda_{u}^{4} / \lambda_{d}^{4}$ can be substantially larger than 1 .

Further qualitative information can be obtained from eqs. (3.7) for the mixing angles. For $i=1,2$, given the very large values of the $\left\langle\hat{Y}_{u}\right\rangle_{i}$, one can expect vanishingly small leftand right-sector mixing angles. For the top case, $i=3$, the left-sector mixing angle is still generally small. In fact, note that the quantities $\Delta_{3}^{u}$ and $\lambda_{u}^{2} v_{R}^{2}+\left(\lambda_{u}^{\prime}\left\langle\hat{Y}_{u}\right\rangle_{3}\right)^{2}$ are very similar in size, and appear with opposite signs in the numerator of $\tan \theta_{L, 3}^{u}$. On the other hand, in the right-sector case, the $\lambda_{u}^{2} v_{R}^{2}$ term appears with reversed sign, and this, depending on the choice of parameters, may result in a non-negligible $\theta_{R, 3}^{u}$.

Figure 5 illustrates the above considerations more quantitatively. The upper panels show the mixing angles in the left-handed top sector against the $M_{W_{R}}$ mass, in the parity (left panel) and in the no-parity case (right panel). One can see that mixing in the lefthanded sector is always fairly small - taking into account the $M_{W_{R}}$ bound discussed in section 3.2, mixing is such that $\sin \left(\theta_{L, 3}^{u}\right) \lesssim 2 \times 10^{-2}$. The corresponding angle in the righthanded sector is displayed in the lower panels for the case of $\mathrm{TeV}$-scale parity (the case of 

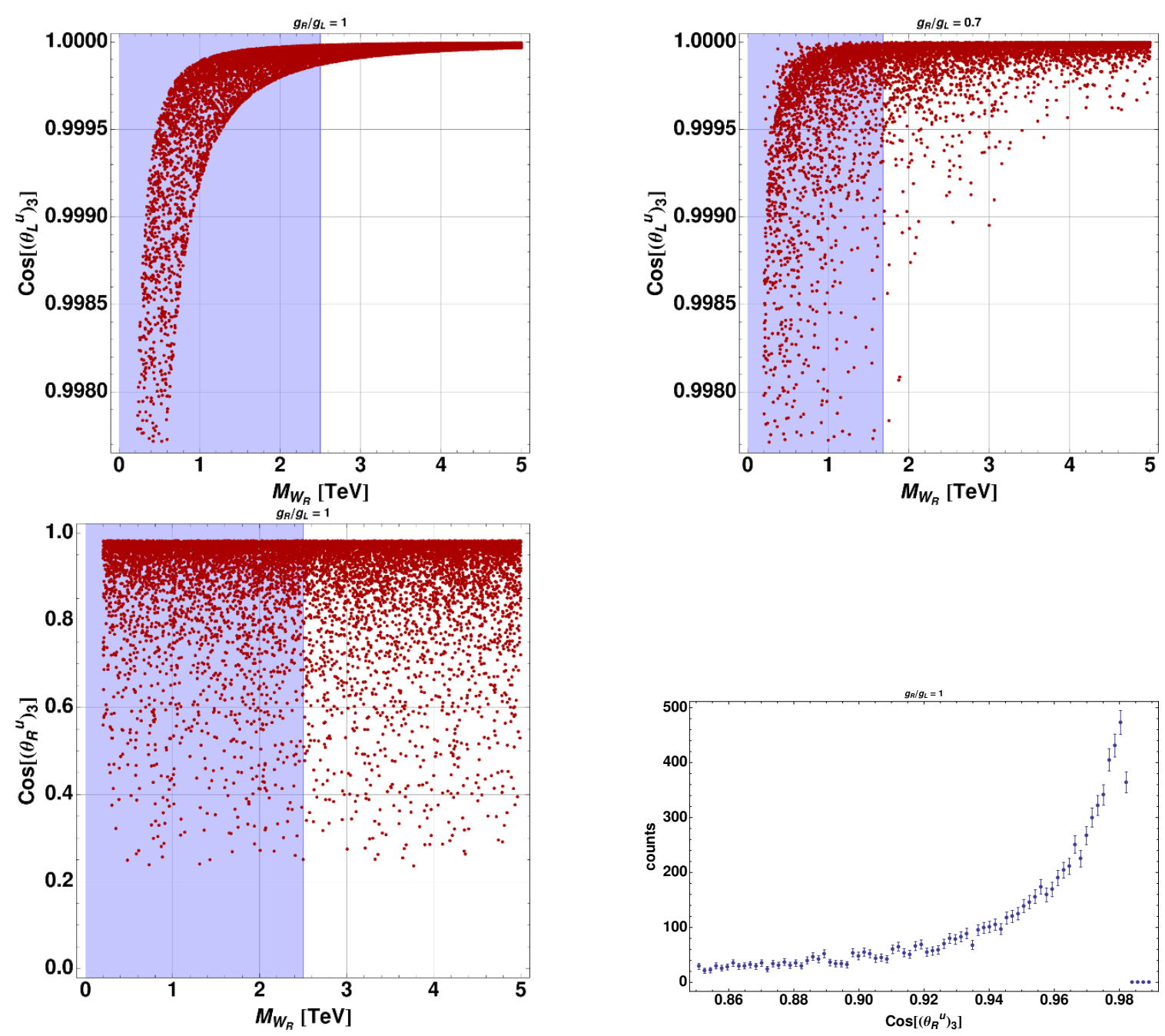

Figure 5. Upper panels: $M_{W_{R}}$ vs. the fermion-mixing angles in the left-handed top sector, for $g_{R} / g_{L}=1$ (leftmost panel) or 0.7 (rightmost panel). Lower panels: $M_{W_{R}}$ vs. the fermion-mixing angles in the right-handed top sector for $g_{R} / g_{L}=1$, and corresponding histogram (see text for further comments).

no parity gives similar results). In this case, the amount of mixing is not affected at all by the $W_{R}$ mass bound, and we typically find $\theta_{R, 3}^{u} \approx 10^{\circ}$ or larger, as also displayed in the histogram. This implies $\sin \left(\theta_{R, 3}^{u}\right) \gtrsim 0.2$, and may lead to potential effects in observables like FCNC top decays such as $t \rightarrow c Z$ and observables sensitive to operators with 4 powers of the top field.

In short, mixing angles anywhere else than in the $t_{R}$ case are vanishingly small. E.g., for $b_{L}$, we find $\sin \left(\theta_{L, 3}^{d}\right) \lesssim 2 \times 10^{-3}$. This bound is relevant to effects in observables like $V_{t b}$ and $R_{b}$, on which we will be more quantitative in section 3.4.1.

\subsubsection{Electroweak precision tests}

$\boldsymbol{Z}^{\mathbf{0}} \rightarrow \boldsymbol{b} \overline{\boldsymbol{b}}$. The decay $Z^{0} \rightarrow b \bar{b}$ is an example of the prototype process $V \rightarrow f \bar{f}$, with $V$ any of the massive vectors, $f$ any of the fermions in the model, and $m_{V} \geq 2 m_{f}$. This kind 
of processes allows to estimate the magnitude of tree-level non-oblique corrections that the model introduces.

The interactions relevant to $Z^{0} \rightarrow b \bar{b}$ are those in $\mathcal{L}_{Z b b} \equiv Z_{\mu}^{0} J_{Z}^{\mu}$, where $J_{Z}^{\mu}$ in our case is as follows

$$
J_{Z}^{\mu}=\frac{g}{c_{w}}\left(\bar{b}_{L} \gamma^{\mu}\left(T_{3}^{d} c_{b_{L}}^{2}-s_{w}^{2} Q_{d}\right) b_{L}+\bar{b}_{R} \gamma^{\mu}\left(-s_{w}^{2} Q_{d}\right) b_{R}\right)+\ldots,
$$

where $T_{3}^{d}$ and $Q_{d}$ are the eigenvalues of the weak isospin $\tau^{3}$ and electric charge operator of down-type quarks, and dots denote the couplings to fermions other than the $b$. For ease of readability, we also abbreviate $\cos \theta_{L(R), 3}^{d}=c_{b_{L(R)}}$ and $\sin \theta_{L(R), 3}^{d}=s_{b_{L(R)}}$. Note that the modification with respect to the SM current is only in the left-handed coupling proportional to $T_{3}^{d}$, because the electric charge operator is diagonal across the quark and heavy-fermion fields, hence it commutes with the rotation (3.5). The relevant Lagrangian (3.11) is entirely analogous to that of ref. [10] hence the correction to $\Gamma\left(Z^{0} \rightarrow b \bar{b}\right)$ in our model will be the same, namely

$$
\frac{\delta \Gamma\left(Z^{0} \rightarrow b \bar{b}\right)}{\Gamma\left(Z^{0} \rightarrow b \bar{b}\right)_{\mathrm{SM}}}=-s_{b_{L}}^{2} \frac{2+4 s_{w}^{2} Q_{d}}{1+4 s_{w}^{2} Q_{d}+8 s_{w}^{4} Q_{d}^{2}}+O\left(s_{b_{L}}^{4}\right)
$$

To get a numerical idea of the correction implied by eq. (3.12), one can first note that, since $s_{w}^{2}$ is a small number, the coefficient of $-s_{b_{L}}^{2}$ is a number close to 2 . Then one can recall, from our previous numerical analysis of fermion mixing, that all the $s_{q_{i}}$ are tiny in the bulk of the model parameter space. In particular, we quoted $s_{b_{L}}^{2} \lesssim 4 \times 10^{-6}$ in the general discussion of fermion mixing. Therefore, the constraint from $Z^{0} \rightarrow b \bar{b}$ plays a completely irrelevant role in our case, in comparison with direct searches of new heavy bosons (we will be back to this later on). In fact, by establishing a lower bound on, e.g., $M_{W_{R}}$, these searches constrain the fermion-mixing angles to have values even closer to zero (see again figure 5).

We note that, from the above argument, the most interesting effects of decays of the kind $V \rightarrow f \bar{f}$ are expected in the top sector, in particular top production and decays.

Oblique corrections. The $S, T$ and $U$ parameters [33] quantify the modifications in the vacuum polarization diagrams for the $\mathrm{SU}(2)_{L} \times \mathrm{U}(1)_{Y}$ vector bosons, due to the fact that the fermionic currents coupled to them are altered with respect to their SM form. In our model, this occurs because of fermionic mixing and because the new fermions have nontrivial charges under $\mathrm{U}(1)_{Y}$. Following customary notation [34], currents are normalized as

$$
J_{a}^{\mu}=\sum_{f} \bar{f} \gamma^{\mu} T^{a} f, \quad J_{Y}^{\mu}=\sum_{f} \bar{f} \gamma^{\mu} Y_{f} f
$$

where $T^{a}$ denotes the $\mathrm{SU}(2)_{L}$ representation, namely the identity or else $\sigma^{a} / 2, Y_{f}$ the hypercharge assignment for fermion $f$, and the sum runs over all fermions reported in the table of section 2 (our fermion definition includes helicity projectors).

The $S$ and $T$ parameters are defined as [33]

$$
S \equiv-\left.16 \pi \Pi_{3 Y}^{\prime}\left(q^{2}\right)\right|_{q^{2}=0}, \quad T \equiv \frac{4 \pi}{s_{w}^{2} c_{w}^{2} M_{Z}^{2}}\left(\Pi_{11}(0)-\Pi_{33}(0)\right)
$$


where $\Pi^{\prime}$ denotes $d \Pi\left(q^{2}\right) /\left.d q^{2}\right|_{q^{2}=0}$ as usual. Their computation is a simple algebraic problem, after defining the 'master' vacuum polarization amplitudes, as the amplitudes with two left-handed currents or respectively one left- and one right-handed current at the two vertices, and fermions of masses $m_{1}, m_{2}$ running in the loop. ${ }^{6}$ These amplitudes are denoted as $\Pi_{L L}\left(m_{1}^{2}, m_{2}^{2}, q^{2}\right)$ and $\Pi_{L R}\left(m_{1}^{2}, m_{2}^{2}, q^{2}\right)$, and we shall follow the definition in [34], that we do not rewrite here explicitly.

For the $T$ parameter we find

$$
\delta T=\frac{3 \pi}{s_{w}^{2} c_{w}^{2} M_{Z}^{2}}\left(-2 s_{t_{L}}^{2} \Pi_{L L}^{b t}+s_{t_{L}}^{2}\left(2-s_{t_{L}}^{2}\right) \Pi_{L L}^{t t}+2 s_{t_{L}}^{2} \Pi_{L L}^{b T}-2 c_{t_{L}}^{2} s_{t_{L}}^{2} \Pi_{L L}^{t T}-s_{t_{L}}^{4} \Pi_{L L}^{T T}\right)
$$

where $\delta T$ indicates that we have subtracted the pure SM contribution, obtained in the limit of no fermion mixing. For ease of readability, we have also denoted $\Pi_{L L}\left(m_{X}, m_{Y}, 0\right)=$ $\Pi_{L L}^{X Y}$, and, again, abbreviated $\cos \theta_{L(R), 3}^{u}=c_{t_{L(R)}}, \sin \theta_{L(R), 3}^{u}=s_{t_{L(R)}}$.

For $\delta S$ we have instead

$$
\begin{aligned}
\delta S=4 \pi\left(-s_{t_{L}}^{2}\left(2-3 s_{t_{L}}^{2}\right) \Pi_{L L}^{\prime t t}+4 s_{t_{L}}^{2} \Pi_{L R}^{\prime t t}+6 c_{t_{L}}^{2} s_{t_{L}}^{2} \Pi_{L L}^{\prime t T}\right. \\
\\
\left.\quad-\left(4 c_{t_{L}}^{2} s_{t_{L}}^{2}+s_{t_{L}}^{4}\right) \Pi_{L L}^{\prime T T}-4 s_{t_{L}}^{2} \Pi_{L R}^{\prime T T}\right) .
\end{aligned}
$$

At this point, we note explicitly that eqs. (3.15) and (3.16) are obtained in the approximation of neglecting fermion mixing other than in the top sector and (this is relevant only for $\delta S$ ) of including in the loops, among the heavy fermions, only the top partner. This is an excellent approximation, given the mass hierarchy among fermionic partners and the size of the mixing angles, discussed before. Both of the $\delta T$ and $\delta S$ corrections turn out to depend only on the LH mixing angle $\theta_{L, 3}^{u}$ - in $\delta T$ the dependence on $\theta_{R, 3}^{u}$ combines in such a way to cancel out in the final result. This fact is very welcome in our case, since, as discussed in section $3.4, \theta_{R, 3}^{u}$ is the only angle sizably different from zero. The allowed experimental values for eqs. (3.15) and (3.16) are of $\mathrm{O}\left(10^{-2}\right)$, with errors of $\mathrm{O}\left(10^{-1}\right)$. Since $s_{t_{L}}^{2}$ is about $10^{-3}$, even for $M_{W_{R}}$ as low as $500 \mathrm{GeV}$, the above corrections, similarly as $\delta \Gamma\left(Z^{0} \rightarrow b \bar{b}\right)$, turn out to play no constraining role at all.

\subsubsection{The decay $\bar{B} \rightarrow X_{s} \gamma$}

Similarly as in [10], a further potential constraint for our model implied by flavor mixing comes from the $\operatorname{BR}\left(\bar{B} \rightarrow X_{s} \gamma\right)$, which is very accurately calculated within the SM [35] and also very precisely measured experimentally [36]. The two figures read, respectively (the photon energy cut is in both cases $E_{\gamma}>1.6 \mathrm{GeV}$.)

$$
\begin{aligned}
\operatorname{BR}\left(\bar{B} \rightarrow X_{s} \gamma\right)_{\exp } & =(3.55 \pm 0.24 \pm 0.09) \times 10^{-4}, \\
\operatorname{BR}\left(\bar{B} \rightarrow X_{s} \gamma\right)_{\mathrm{SM}, \mathrm{NNLO}} & =(3.15 \pm 0.23) \times 10^{-4},
\end{aligned}
$$

showing very good agreement with each other.

\footnotetext{
${ }^{6}$ Specifically, a left- or a right-handed current means an insertion of $i \gamma^{\mu} \frac{\left(1 \mp \gamma^{5}\right)}{2}$ at the vertex, with namely no other overall factor involved, e.g. color factors.
} 
This decay in the SM is generated by a 'magnetic-penguin' operator induced by a $W-t_{L}$ loop. Its Wilson coefficient at the $W$ scale, $C_{7}\left(m_{t}, M_{W}\right)$, is modified in our model because the $t_{L}$ is not a mass eigenstate: $t_{L}=c_{t_{L}} \hat{t}_{L}+s_{t_{L}} \hat{\psi}_{L}^{t}$ (we have again used the shortcut $\left.\cos \left(\theta_{L, 3}^{u}\right)=c_{t_{L}}\right)$. Neglecting the running between the $\psi^{t}$ mass, here indicated as $m_{T}(\gtrsim 500 \mathrm{GeV}$, as we discussed in section 3.4) and the $W$ scale, this effect can be accounted for by a shift in $C_{7}\left(m_{t}, M_{W}\right)$,

$$
C_{7}\left(m_{t}, M_{W}\right) \rightarrow c_{L}^{2} C_{7}\left(m_{t}, M_{W}\right)+s_{L}^{2} C_{7}\left(m_{T}, M_{W}\right),
$$

plus an analogous shift in the coefficient $C_{8}\left(m_{t}, M_{W}\right)$ of the chromomagnetic penguin operator. Since $C_{7}\left(\mu \approx m_{b}\right)$ enters as $\left|C_{7}\right|^{2}$ in the branching ratio, the leading effect is due to interference, and is of $\mathrm{O}\left(s_{t_{L}}^{2}\right)$. To get a numerical idea of the effect, one may use the next-to-leading order (NLO) SM formulae of [37]. Including the shift (3.18) and using $M_{T}=500 \mathrm{GeV}$, we obtain

$$
\operatorname{BR}\left(\bar{B} \rightarrow X_{s} \gamma\right)=\left(3.2+1.3 s_{t_{L}}^{2}\right) \times 10^{-4}
$$

In view of the smallness of $s_{t_{L}}^{2}$ in the bulk of our parameter space, the above shift is well within the theoretical error.

\subsection{Further constraints}

\subsubsection{Electric dipole moments}

After diagonalizing the quark - heavy-fermion mass matrix, all CP violating fermion couplings arise from the $Y_{u}$ or $Y_{d}$ vevs. In particular, with our choice of basis in eq. (2.6), they must be proportional to $\left\langle Y_{u}\right\rangle$. One may expect that one-loop diagrams with intermediate gauge bosons (either $W_{R}$ or the flavor bosons $\mathcal{G}_{i}$ ) and up quarks, and one quark mass insertion, may result in new contributions to the up quark EDM. In the flavor-boson case, using eq. (2.5), it is however easy to convince oneself that the contribution to the EDM must be of the form

$$
d_{e}^{u} \propto \frac{\lambda_{u}^{2} v_{L} v_{R}}{\lambda^{\prime}} \operatorname{Im}\left(\frac{\lambda^{a}}{2} V_{\mathrm{CKM}}^{\dagger}\left\langle\hat{Y}_{u}\right\rangle^{-1} V_{\mathrm{CKM}} \frac{\lambda^{a}}{2}\right)_{11},
$$

with $\lambda^{a}$ the Gell-Mann matrices. Similarly as the one-loop SM contribution, the contribution in eq. (3.20) vanishes trivially because of the hermiticity of the matrix on the r.h.s.. A completely similar argument holds of course in the $W_{R}$ case. Hence new contributions to quark EDMs may arise in our model only at the two-loop level and are therefore very small.

\subsubsection{Top quark flavor changing effects}

Among the model predictions testable at the LHC are top-quark flavor changing effects, e.g. a modification in the $\bar{t} c G$ coupling. In our model, neutral Higgs interactions do not give rise to any flavor changing effect due to the fact that they are diagonal. However, the flavor gauge boson couplings involve the CKM matrix as well as the flavor generators, both of which can mix generations. We will do a detailed study of these effects in a subsequent 
paper. Here we simply give an estimate of the dominant contribution to the operator $\bar{t} \sigma_{\mu \nu} c G^{\mu \nu}$ to be of order

$$
g_{t c G} \sim \frac{v_{L} v_{R}}{16 \pi^{2}\left(\left\langle\hat{Y}_{u}\right\rangle_{3}\right)^{3}}
$$

which can be estimated to be of order $10^{-3}\left(\frac{\mathrm{TeV}}{\left\langle\hat{Y}_{u}\right\rangle_{3}}\right)^{3} \mathrm{TeV}^{-1}$. Such effects have been looked for at the Tevatron and will be looked for in processes such as $G G \rightarrow t \bar{c}, c G \rightarrow t \gamma$, etc. at the LHC $[38,39]$. The current Tevatron (DØ) bound on the strength of such operators is $\leq 0.018 \mathrm{TeV}^{-1}$ with a $2.2 \mathrm{fb}^{-1}$ dataset [40].

\subsubsection{Direct searches}

A key feature of models of this kind is the existence of three heavy vectorlike families, which essentially helps to ameliorate the severe FCNC bounds expected on the basis of dimensional analysis. In this section we address the bounds on their masses based on direct collider searches. The CDF collaboration has searched for up-type heavy quarks (called generically $t^{\prime}$ in the literature) and provides a lower bound on their mass of $335 \mathrm{GeV}$ [41, 42]. Likewise, there is a lower limit on down-type heavy quarks, also from CDF, giving $m_{\psi_{d}} \geq$ $385 \mathrm{GeV}[43,44]$. These analyses assume the heavy quarks to decay $100 \%$ of the time to a $W$ and light quarks. This will hold in our model for the lightest of the vectorlike quarks.

\section{Lepton sector}

Within our framework, the discussion of the lepton sector is completely parallel to the quark sector as far as the flavor gauge boson and charged lepton spectra are concerned. The relevant flavor gauge group is in this case $\mathrm{SU}(3)_{\ell_{L}} \times \mathrm{SU}(3)_{\ell_{R}}$, and one introduces two further flavon fields $Y_{\nu, \ell}$, transforming as $(\overline{3}, 3)$ under this group. The gauge invariant Yukawa interaction for the leptons is then completely analogous to eq. (2.1), but for the replacement of quark doublets with lepton ones and heavy quark partners with heavy lepton partners. Of course, the $\lambda$ and $\lambda^{\prime}$ couplings also do not need to be the same as those appearing in eq. (2.1). The fermion mixing argument leading to eq. (2.7) is likewise trivially generalizable to this case, hence for the diagonal elements of $\left\langle Y_{\ell}\right\rangle$ one expects the relation $\left\langle\hat{Y}_{e}\right\rangle:\left\langle\hat{Y}_{\mu}\right\rangle:\left\langle\hat{Y}_{\tau}\right\rangle=m_{e}^{-1}: m_{\mu}^{-1}: m_{\tau}^{-1}$.

\subsection{Neutrino masses}

Concerning the neutrino sector, after symmetry breaking the mass matrix for $\left(\nu_{L, R}, \psi_{L, R}^{\nu}\right)$ separates into two block matrices involving $\left(\nu_{L}, \psi_{R}^{\nu}\right)$ or $\left(\nu_{R}, \psi_{L}^{\nu}\right)$. For the first case we have

$$
M_{\nu-N}=\left(\begin{array}{cc}
0 & \lambda_{\nu} v_{L} \\
\lambda_{\nu} v_{R} & \left\langle Y_{\nu}\right\rangle
\end{array}\right)
$$

and similarly for the $\left(\nu_{R}, \psi_{L}^{\nu}\right)$, after exchanging $L \leftrightarrow R$ in the above matrix. As a result, we have two sets of Dirac neutrinos: $\nu_{L}$ pairing with $\psi_{R}^{\nu}$ and $\nu_{R}$ with $\psi_{L}^{\nu}$. In the limit of 
$\left\langle Y_{\nu}\right\rangle \gg v_{R}$, the neutrino mass formula reads

$$
M_{\nu}=\frac{\lambda_{\nu}^{2} v_{L} v_{R}}{\left\langle Y_{\nu}\right\rangle}
$$

It is clear from the above equation that, if $v_{R}$ and $\left\langle Y_{\nu}\right\rangle$ are in the few $\mathrm{TeV}$ range, we need to choose $\lambda_{\nu} \sim 10^{-6}$ in order to get the right order of magnitude for neutrino masses (in the sub-eV range). Note that already this is an improvement over the SM, where getting Dirac masses of the right order requires the Yukawa coupling to be much smaller (of order $\left.10^{-12}\right)$. Furthermore, we need to choose $\left\langle Y_{\nu}\right\rangle$ in such a way as to get the observed large neutrino mixings. As far as the $\psi_{L, R}^{\nu}$ fields are concerned they will have masses of order of the flavor symmetry breaking scale $\left\langle Y_{\nu}\right\rangle$.

\subsection{Constraints}

The above setup is subject to various constraints. First, since neutrinos are Dirac fermions, the right-handed neutrinos have $W_{R}$-mediated interactions, that can keep them in equilibrium with charged leptons, unless the right-handed interactions are sufficiently weak. Therefore, the model will predict $N_{\nu}=6$ at the Big Bang Nucleosynthesis epoch, which is not consistent with our current understanding of Helium, Deuterium and Lithium abundances of the universe [45]. In fact, this leads to a lower bound on the mass of the righthanded $W_{R}$ 's of order $3.3 \mathrm{TeV}$ [46]. It must however be noted that, if one generates Majorana masses for the $\psi_{R}^{\nu}$ by adding $\mathrm{SU}(3)_{R}$ sextet Higgs fields with vev, one can lift the right-handed neutrinos to higher masses and understand the lightness of left-handed neutrino masses via the seesaw mechanism. In this case, there is no lower bound on the $W_{R}$ mass from Big Bang Nucleosynthesis.

Second, it is interesting to note a recent lower bound on the $W_{R}$ mass of $1.36 \mathrm{TeV}$ from the CMS experiment at the LHC [47]. This bound directly applies to our model, and in general to models with Dirac neutrinos.

Third, within this generalization of the model, possible constraints on the flavor gauge boson scale may come from lepton-flavor violating (LFV) decays such as $\mu \rightarrow e \gamma$ and $\mu \rightarrow 3 e$. The existing limits on these decays can actually be used to estimate a lower bound on the leptonic flavor scale as follows. Formula (4.2) for the Dirac neutrino mass matrix can be trivially inverted to give

$$
\left\langle Y_{\nu}\right\rangle=\frac{\lambda_{\nu}^{2} v_{L} v_{R}}{M_{\nu}}
$$

This expression can then be rewritten using the formula $M_{\nu}=U^{*} \hat{M}_{\nu} U^{\dagger}$ (where $\hat{M}_{\nu}$ is the diagonal neutrino mass matrix) as

$$
\left\langle Y_{\nu}\right\rangle_{\alpha \beta}=\lambda_{\nu}^{2} v_{L} v_{R} \sum_{i} U_{\alpha i}^{*} U_{\beta i}^{*} m_{i}^{-1}
$$

with $m_{i}$ the diagonal entries of $\hat{M}_{\nu}$. The form of $\left\langle Y_{\nu}\right\rangle$ clearly depends on the neutrino mass ordering. Taking for simplicity normal ordering, $m_{1} \ll m_{2} \ll m_{3}$, we get the dominant contribution to be

$$
\left\langle Y_{\nu}\right\rangle_{\alpha \beta}=\lambda_{\nu}^{2} v_{L} v_{R} U_{\alpha 1}^{*} U_{\beta 1}^{*} m_{1}^{-1}
$$


To have an estimate of the typical $\left\langle Y_{\nu}\right\rangle$ size, one may choose $m_{1} \sim 0.5 m_{\odot} \sim 0.005 \mathrm{eV}$ and use the tri-bi-maximal form for the lepton mixing matrix $U$. For a $\mathrm{TeV} v_{R}$, we find $\left\langle Y_{\nu}\right\rangle$ entries of $\sim 100 \mathrm{TeV}$.

We can now provide an estimate of the decay rates for the processes $\mu \rightarrow 3 e$ and $\mu \rightarrow e \gamma$. The amplitude for the $\mu \rightarrow 3 e$ process arises at the tree level due to flavor diagonal and off-diagonal gauge boson mixing, namely from the terms $\left\langle Y_{\nu}\right\rangle_{11}$ and $\left\langle Y_{\nu}\right\rangle_{12}$. Since neutrino mixings are large, we assume these terms to be of similar size, indicated as $\bar{Y}_{\nu}$. Hence the amplitude has the form

$$
A(\mu \rightarrow 3 e) \sim \frac{1}{2 \bar{Y}_{\nu}^{2}} .
$$

This relation is nothing but a simplified version of eqs. ( 3.2). Note, in particular, that the gauge coupling dependence is of course absent, because the flavor-gauge boson masses also scale with it. To translate eq. (4.6) into a branching ratio, one can use the fact that the calculation of $\mu \rightarrow 3 e$ is very similar to the well-known calculation of $\Gamma\left(\mu \rightarrow e \nu_{\mu} \bar{\nu}_{e}\right) \simeq \Gamma_{\mu \text {,tot }}$, but for the replacement of $G_{F} / \sqrt{2}$ with the amplitude in eq. (4.6). Hence the branching ratio for $\mu \rightarrow 3 e$ can be simply estimated as

$$
B(\mu \rightarrow 3 e) \sim \frac{1}{2 \bar{Y}_{\nu}^{4} G_{F}^{2}} .
$$

This can be of order $10^{-12}$, like the current experimental limit [48], for $\bar{Y}_{\nu} \sim 300 \mathrm{TeV}$. Because of our assumption of roughly equal entries in the $Y_{\nu}$ vev matrix, the same estimate applies to all the other LFV decays into three charged leptons, such as $\tau \rightarrow 3 e$ or $\tau \rightarrow 3 \mu$. The limits on these decays are (currently) much weaker $[49,50]$ and as such satisfied for the above mentioned value of the leptonic flavor scale. The situation may of course change drastically in the event of new data from a super flavor factory.

Turning to the $\mu \rightarrow e \gamma$ decay, it is generated by a loop graph, and its branching ratio can be estimated to be of order $\frac{27 \alpha}{16 \pi G_{F}^{2} \bar{Y}_{\nu}^{4}}$. Given the loop suppression with respect to eq. (4.7), one gets values safely below the current experimental limits [51] for the above choice $\bar{Y}_{\nu} \sim 300 \mathrm{TeV}$.

A final comment is in order. The above discussion about LFV observables was mostly aimed at verifying that reasonable values for the relevant massive parameters of the model do not lead to conflicts with the current LFV bounds. A separate and potentially interesting question not addressed in this paper is whether our setup may explain a positive LFV signal from current or planned experiments. While our arguments, in particular the one following eq. (4.7), suggest a positive answer, a more detailed one requires invoking a specific flavor model to be embedded within our framework.

\section{Conclusions}

We have examined the possibility of gauged flavor symmetry as a way to explore the origin of quark lepton masses and mixings. As was noted in ref. [10], in such models there is an inverse correlation between the quark masses and the flavor hierarchy between the gauge 
boson masses, making it possible to have light enough flavor gauge bosons and enhanced FCNC effects for the third generation. We have worked within the left-right symmetric electroweak group, which seems to provide a number of advantages over the SM gauge group while maintaining this inverse relation. These advantages include a reduction in the number of input parameters, a possible solution to the strong CP problem without the axion (provided parity is also a $\mathrm{TeV}$-scale symmetry), and the possibility of accommodating neutrino masses. For the case where parity is a $\mathrm{TeV}$-scale symmetry, the lower bounds on both the lightest vectorlike fermion mass as well as on the flavor gauge symmetry scale is of about 5 and respectively $10 \mathrm{TeV}$ (see figures 4 and 3). On the other hand, if only $\mathrm{SU}(2)_{R}$, but not parity, survives as a good symmetry down to the $\mathrm{TeV}$ scale, the lightest phenomenologically allowed vectorlike quark mass could be much lower. The lightest flavor gauge boson mass gets likewise lower. How low one can go down for these masses depends on what one assumes for the difference between the left and the right couplings, which in turn depends on the nature of the UV complete parity-symmetric theory. We have noted the consistency of the model with all the best-known phenomenology, including electroweak precision data. The detailed predictions for the FCNC effects in the third generation case are currently under investigation; here we only made some qualitative comments about top flavor changing effects.

\section{Acknowledgments}

DG acknowledges useful discussions with Roberto Contino, Adam Falkowski, Slava Rychkov and Giovanni Villadoro. The authors also acknowledge Giovanni Villadoro for various comments on the manuscript. The work of DG was supported by the EU Marie Curie IEF Grant no. PIEF-GA-2009-251871. The work of RNM was supported by the NSF grant PHY-0968854, and the work of IS was supported by the U.S. Department of Energy through grant DE-FG02-93ER-40762.

Open Access. This article is distributed under the terms of the Creative Commons Attribution Noncommercial License which permits any noncommercial use, distribution, and reproduction in any medium, provided the original author(s) and source are credited.

\section{References}

[1] F. Wilczek, Axions and family symmetry breaking, Phys. Rev. Lett. 49 (1982) 1549 [SPIRES].

[2] R.N. Mohapatra, Gauge model for chiral symmetry breaking and muon electron mass ratio, Phys. Rev. D 9 (1974) 3461 [SPIRES].

[3] S.M. Barr and A. Zee, Calculating the electron mass in terms of measured quantities, Phys. Rev. D 17 (1978) 1854 [SPIRES].

[4] C.L. Ong, Adding a horizontal gauge symmetry to the Weinberg-Salam model: an eight quark model, Phys. Rev. D 19 (1979) 2738 [SPIRES].

[5] F. Wilczek and A. Zee, Horizontal interaction and weak mixing angles, Phys. Rev. Lett. 42 (1979) 421 [SPIRES]. 
[6] J. Chakrabarti, Horizontal gauge symmetry and a new picture for the $b$ quark, Phys. Rev. D 20 (1979) 2411 [SPIRES].

[7] T. Maehara and T. Yanagida, Gauge symmetry of horizontal flavor, Prog. Theor. Phys. 61 (1979) 1434 [SPIRES].

[8] A. Davidson, M. Koca and K.C. Wali, U(1) as the minimal horizontal gauge symmetry, Phys. Rev. Lett. 43 (1979) 92 [SPIRES].

[9] J. Chakrabarti, M. Popovic and R. N. Mohapatra, Problem of fermion generations in grand unified theories, Phys. Rev. D 21 (1980) 3212 [SPIRES].

[10] B. Grinstein, M. Redi and G. Villadoro, Low scale flavor gauge symmetries, JHEP 11 (2010) 067 [arXiv: 1009.2049] [SPIRES].

[11] A. Davidson and K.C. Wali, Family mass hierarchy from universal seesaw mechanism, Phys. Rev. Lett. 60 (1988) 1813 [SPIRES].

[12] K.S. Babu and R.N. Mohapatra, CP violation in seesaw models of quark masses, Phys. Rev. Lett. 62 (1989) 1079 [SPIRES].

[13] K.S. Babu and R.N. Mohapatra, A solution to the strong CP problem without an axion, Phys. Rev. D 41 (1990) 1286 [SPIRES].

[14] J.C. Pati and A. Salam, Lepton number as the fourth color, Phys. Rev. D 10 (1974) 275 [SPIRES].

[15] R.N. Mohapatra and J.C. Pati, Left-right gauge symmetry and an isoconjugate model of CP-violation, Phys. Rev. D 11 (1975) 566 [SPIRES].

[16] R.N. Mohapatra and J.C. Pati, A natural left-right symmetry, Phys. Rev. D 11 (1975) 2558 [SPIRES].

[17] G. Senjanović and R.N. Mohapatra, Exact left-right symmetry and spontaneous violation of parity, Phys. Rev. D 12 (1975) 1502 [SPIRES].

[18] M.A.B. Beg and H.S. Tsao, Strong p, $t$ noninvariances in a superweak theory, Phys. Rev. Lett. 41 (1978) 278 [SPIRES].

[19] R.N. Mohapatra and G. Senjanović, Natural suppression of strong $p$ and $t$ noninvariance, Phys. Lett. B 79 (1978) 283 [SPIRES].

[20] K.S. Babu and R.N. Mohapatra, A solution to the strong CP problem without an axion, Phys. Rev. D 41 (1990) 1286 [SPIRES].

[21] D. Chang, R.N. Mohapatra and M.K. Parida, Decoupling parity and SU(2)-R breaking scales: a new approach to left-right symmetric models, Phys. Rev. Lett. 52 (1984) 1072 [SPIRES].

[22] W.-M. Yang and H.-H. Liu, The new extended left-right symmetric grand unified model with SO(3) family symmetry, Nucl. Phys. B 820 (2009) 364 [arXiv:0901.0233] [SPIRES].

[23] W.-M. Yang, Fermion masses and flavor mixings from family symmetry $\mathrm{SU}(3)$, arXiv: 1011.4573 [SPIRES].

[24] D. Bhowmick, A.K. Ray and S. Raychaudhuri, CP-violating parameters in a left-right symmetric model with an $\mathrm{SU}(3)_{V L} H$ horizontal symmetry, Int. J. Mod. Phys. A 13 (1998) 3799 [SPIRES].

[25] T. Feldmann, See-saw masses for quarks and leptons in SU(5), arXiv:1010.2116 [SPIRES]. 
[26] UTfit collaboration, M. Bona et al., Model-independent constraints on $\Delta F=2$ operators and the scale of new physics, JHEP 03 (2008) 049 [arXiv:0707.0636] [SPIRES].

[27] G. Beall, M. Bander and A. Soni, Constraint on the mass scale of a left-right symmetric electroweak theory from the $K_{L} K_{S}$ mass difference, Phys. Rev. Lett. 48 (1982) 848 [SPIRES].

[28] R.N. Mohapatra, G. Senjanović and M.D. Tran, Strangeness changing processes and the limit on the right-handed gauge boson mass, Phys. Rev. D 28 (1983) 546 [SPIRES].

[29] K. Kiers, J. Kolb, J. Lee, A. Soni and G.-H. Wu, Ubiquitous CP-violation in a top inspired left-right model, Phys. Rev. D 66 (2002) 095002 [hep-ph/0205082] [SPIRES].

[30] Y. Zhang, H. An, X. Ji and R.N. Mohapatra, Right-handed quark mixings in minimal left-right symmetric model with general CP-violation, Phys. Rev. D 76 (2007) 091301 [arXiv: 0704.1662] [SPIRES].

[31] Y. Zhang, H. An, X. Ji and R.N. Mohapatra, General CP-violation in minimal left-right symmetric model and constraints on the right-handed scale, Nucl. Phys. B 802 (2008) 247 [arXiv: 0712.4218] [SPIRES].

[32] A. Maiezza, M. Nemevsek, F. Nesti and G. Senjanović, Left-right symmetry at LHC, Phys. Rev. D 82 (2010) 055022 [arXiv:1005.5160] [SPIRES].

[33] M.E. Peskin and T. Takeuchi, Estimation of oblique electroweak corrections, Phys. Rev. D 46 (1992) 381 [SPIRES].

[34] M. E. Peskin and D. V. Schroeder, An introduction to quantum field theory, Addison Wesley, Reading U.S.A. (1995).

[35] M. Misiak et al., The first estimate of $B(\bar{B} \rightarrow X / s \gamma)$ at $O\left(\alpha_{s}^{2}\right)$, Phys. Rev. Lett. 98 (2007) 022002 [hep-ph/0609232] [SPIRES].

[36] Heavy Flavor Averaging Group collaboration, D. Asner et al., Averages of b-hadron, c-hadron and $\tau$-lepton Properties, arXiv:1010.1589 [SPIRES].

[37] K.G. Chetyrkin, M. Misiak and M. Münz, Weak radiative B-meson decay beyond leading logarithms, Phys. Lett. B 400 (1997) 206 [hep-ph/9612313] [SPIRES].

[38] R.A. Coimbra et al., Dimension six FCNC operators and top production at the LHC, Phys. Rev. D 79 (2009) 014006 [arXiv:0811.1743] [SPIRES].

[39] T. Han, K. Whisnant, B.L. Young and X. Zhang, Searching for $t \rightarrow c g$ at the Fermilab Tevatron, Phys. Lett. B 385 (1996) 311 [hep-ph/9606231] [SPIRES].

[40] D0 collaboration, V.M. Abazov et al., Search for flavor changing neutral currents via quark-gluon couplings in single top quark production using $2.3 \mathrm{fb}^{-1}$ of ppbar collisions, Phys. Lett. B 693 (2010) 81 [arXiv: 1006.3575] [SPIRES].

[41] CDF collaboration, A. Lister, Search for heavy top-like quarks $t^{\prime} \rightarrow W q$ using lepton plus jets events in $1.96 \mathrm{TeV}$ p $\bar{p}$ collisions, arXiv:0810.3349 [SPIRES].

[42] CDF collaboration, J. Conway et al., Search for heavy top $t^{\prime} \rightarrow W q$ in lepton plus jets events in $\int \mathcal{L} d t=4.6 f^{-1}, \mathrm{CDF} / \mathrm{PUB} / \mathrm{TOP} / \mathrm{PUBLIC} / 10110$.

[43] CDF collaboration, L. Scodellaro, Searches for $W^{\prime}$ and $b^{\prime}$ at the Tevatron, talk given at $35^{\text {th }}$ Conference on High Energy Physics (ICHEP2010), July 22-28, Paris, France (2010).

[44] D. Whiteson t al., Search for heavy bottom-like chiral quarks decaying to an electron or muon and jets, CDF/PHYS/EXO/PUBLIC/10243. 
[45] Particle Data Group collaboration, K. Nakamura, Review of particle physics, J. Phys. G 37 (2010) 075021.

[46] R.H. Cyburt, B.D. Fields, K.A. Olive and E. Skillman, New BBN limits on physics beyond the standard model from He4, Astropart. Phys. 23 (2005) 313 [astro-ph/0408033] [SPIRES].

[47] CMS collaboration, V. Khachatryan et al., Search for a heavy gauge boson $W^{\prime}$ in the final state with an electron and large missing transverse energy in pp collisions at $\sqrt{s}=7 \mathrm{TeV}$, Phys. Lett. B 698 (2011) 21 [arXiv:1012.5945] [SPIRES].

[48] SINDRUM collaboration, U. Bellgardt et al., Search for the decay $\mu^{+} \rightarrow e^{+} e^{+} e^{-}$, Nucl. Phys. B 299 (1988) 1 [SPIRES].

[49] K. Hayasaka et al., Search for lepton flavor violating tau decays into three leptons with 719 million produced $\tau^{+} \tau^{-}$pairs, Phys. Lett. B 687 (2010) 139 [arXiv:1001.3221] [SPIRES].

[50] BABAR collaboration, J.P. Lees et al., Limits on $\tau$ lepton-flavor violating decays in three charged leptons, Phys. Rev. D 81 (2010) 111101 [arXiv:1002.4550] [SPIRES].

[51] MEGA collaboration, M. Ahmed et al., Search for the lepton-family-number nonconserving decay $\mu \rightarrow e+\gamma$, Phys. Rev. D 65 (2002) 112002 [hep-ex/0111030] [SPIRES]. 